PALEO

Revue d'archéologie préhistorique

19 | 2007

Spécial table ronde ( 1 ère partie) : Le Gravettien : entités régionales d'une paléoculture européenne, Les Eyzies, juillet 2004

\title{
Cadre stratigraphique et chronologique du Gravettien en Europe centrale
}

Stratigraphy and chronological context of the Gravettian in Central Europe

Paul Haesaerts, Ilie Borziac, Vasile Chirica, Freddy Damblon et Larissa Koulakovska

\section{OpenEdition}

Journals

Édition électronique

URL : https://journals.openedition.org/paleo/496

DOI : $10.4000 /$ paleo.496

ISSN : 2101-0420

Éditeur

SAMRA

Édition imprimée

Date de publication : 30 décembre 2007

Pagination : 31-51

ISSN : 1145-3370

Référence électronique

Paul Haesaerts, Ilie Borziac, Vasile Chirica, Freddy Damblon et Larissa Koulakovska, « Cadre

stratigraphique et chronologique du Gravettien en Europe centrale », PALEO [En ligne], 19 | 2007, mis en ligne le 21 octobre 2009, consulté le 26 juin 2021. URL : http://journals.openedition.org/paleo/496 ; DOI : https://doi.org/10.4000/paleo.496

\section{(C)}

PALEO est mis à disposition selon les termes de la licence Creative Commons Attribution - Pas d'Utilisation Commerciale - Pas de Modification 4.0 International. 


\title{
CADRE STRATIGRAPHIQUE ET CHRONOLOGIQUE DU GRAVETTIEN EN EUROPE CENTRALE
}

\author{
Paul HAESAERTS ${ }^{(1)}$, Ilie BORZIAC (2), Vasile CHIRICA ${ }^{(3)}$, \\ Freddy DAMBLON ${ }^{(1)}$, Larissa KOULAKOVSKA ${ }^{(4)}$
}

\begin{abstract}
Résumé : Dans le bassin du Danube moyen, les sites de Willendorf II (Autriche), de Dolní Vestonice, de Pavlov et de Stránská Skála (République tchèque) ont permis de reconstruire une séquence régionale bien documentée pour les loess du pléniglaciaire moyen (de \pm 45000 à 26000 BP), tandis que le contrôle chronologique pour le pléniglaciaire supérieur et le Tardiglaciaire (de 26000 à 10000 BP) restait limité en raison du faible nombre de gisements du Paléolithique supérieur relevant de cette période. Depuis 1991, de nouvelles données obtenues dans la région est-carpatique (bassins du Prut moyen et du Dniestr moyen) sont basées sur trois enregistrements loessiques exceptionnels : Mitoc-Malu Galben (Roumanie), Cosautsi (République de Moldavie) et Molodova (Ukraine). Pour la période comprise entre 33000 et $10000 \mathrm{BP}$, plus de 15 oscillations climatiques positives marquées par des sols humifères en alternance avec des loess et des sols cryogéniques ont été enregistrées et positionnées chronologiquement sur la base de plus de 120 nouvelles datations ${ }^{14} \mathrm{C}$ réalisées dans les laboratoires de Groningen et d'Oxford. L'ensemble des données issues des deux régions ouest- et est-carpatique a fourni une séquence globale au niveau paléoclimatique, chronologique et archéologique conduisant à une meilleure compréhension des changements de l'environnement par rapport aux témoins matériels du Paléolithique supérieur à l'échelle de l'Europe centrale.
\end{abstract}

Mots-clés : Loess, Europe centrale, Gravettien, stratigraphie, chronologie.

Abstract: Stratigraphy and chronological context of the Gravettian in Central Europe. In the Middle Danube basin, the sites of Willendorf I (Austria), Dolní Vestonice, Pavlov and Stránská Skála (Czech Republic) allowed the reconstruction of a well documented regional loess sequence for the middle pleniglacial $( \pm 45,000$ to $26,000 \mathrm{BP}$ ), but the chronological setting of the late pleniglacial and late glacial (26,000 to 10,000 BP) remained limited due to the low amount of Upper Palaeolithic sites of this period. Since 1991, new data obtained in the East Carpathian Area (Middle Prut and Middle Dniester basins) are based on three well documented local loess records: Mitoc-Malu Galben (Romania), Cosautsi (Republic of Moldova) and Molodova (Ukraine). In the time-span between 33,000 and 10,000 BP, more than 15 positive climatic oscillations marked by humiferous soils in alternation with loess and cryogenic soils were recorded and chronologically positioned on the ground of some 120 new radiocarbon dates from Groningen and Oxford laboratories. The whole data from both West and East Carpathian areas provided a global palaeoclimatic, chronological and archaeological sequence allowing a better understanding of the environmental changes with regard to the Upper Palaeolithic occurrences at the scale of Central Europe.

Key-words: Loess, Central Europe, Gravettian, stratigraphy, chronology.

\section{1 - INTRODUCTION}

En Europe centrale, la majorité des gisements de plein air du Paléolithique supérieur se répartissent dans deux domaines géographiques de part et d'autre de la chaîne des Carpates (fig. 1). Le domaine occidental couvre une large part du bassin du Danube moyen ; les gisements y sont bien documentés en Basse Autriche, dans la vallée du Váh en Slovaquie occidentale et le long du couloir morave qui donne accès vers le nord à la plaine baltique. Dans le domaine oriental, ils sont surtout abondants le long du Prut et du Dniestr qui drainent les plateaux loessiques de Podolie et de Moldavie et entaillent les formations riches en silex du Crétacé supérieur.

(1) Institut royal des Sciences naturelles de Belgique, rue Vautier 29, B-1000 Bruxelles, Belgique paul.haesaerts@naturalsciences.be

(2) Institut d'Archéologie, Académie des Sciences, PI. Stefan cel Mare 1, 277612 Kishinev, République de Moldavie (3) Institut d'Archéologie de l'Académie de Roumanie, Branche de Jassy, rue L. Catargiu 18, 6600 Jassy, Roumanie vchirica@yahoo.com

(4) Institut d'Archéologie, 12 avenue des Héros de Stalingrad, 04210 Kiev-210, Ukraine 


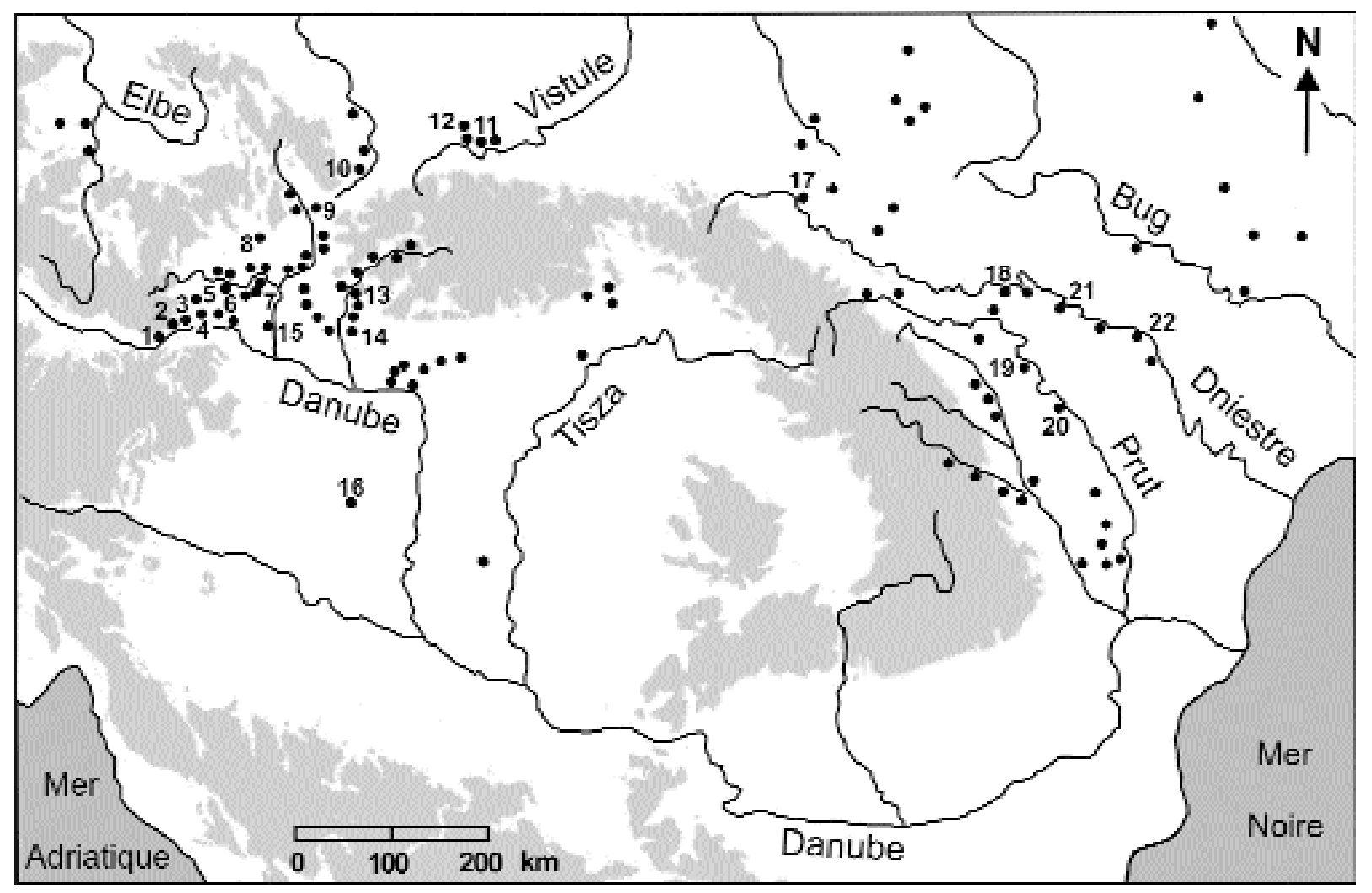

Figure 1 - Situation des principaux gisements gravettiens.

Figure 1 - Location of the main Gravettian sites.

1: Aggsbach et Willendorf; 2: Krems-Hundssteig; 3: Stratzing-Galgenberg; 4: Grubgraben et Langelois ; 5: Kamegg; 6: Alberndorf; 7: Dolní Vestonice, Pavlov et Milovice; 8: Stránská Skála; 9: Predmostí; 10: Petrkovice; 11: Spadzista; 12: Bzroskvinia; 13: Moravany; 14: Nitra Cermán; 15: Stillfried; 16: Sagvar; 17: Mejigirzi; 18: Molodova; 19: Mitoc-Malu Galben et Crasnaleuca; 20: Ripiceni-Izvor; 21: Cosautsi et Podgor; 22: Climautsi.

Au cours des années soixante, les enregistrements de Stillfried B à l'est de Vienne (Fink 1969) et de Dolní Vestonice dans le sud de la Moravie (Klíma 1963 ; Demek \& Kukla 1969) constituèrent la référence pour la seconde moitié du Pléistocène supérieur en Europe centrale. La stratigraphie du système se composait d'un sol humifère daté vers $29000 \mathrm{BP}$ et rapporté au pléniglaciaire moyen (sol de Stillfried B en Autriche, PK I et sol Würm 2/3 en Moravie), surmonté par un ensemble de loess à pseudogleys rapporté au pléniglaciaire supérieur, surtout bien développé à Dolní Vestonice.

Pendant les années quatre-vingt, des stratigraphies complémentaires bien documentées et datées par le ${ }^{14} \mathrm{C}$ furent établies pour des gisements paléolithiques pluristratifiés répartis dans la vallée du Danube aux environs de Krems (notamment à Willendorf Stratzing et Grubgraben), aux environs de Brno (Bohunice et Stránská Skála) et dans le sud de la Pologne (Spadzista). Parallèlement, en Ukraine occidentale, les remarquables séquences de Molodova et de Korman furent mises au jour dans la vallée du Dniestr grâce aux travaux de A. Chernysh (1959) et de I. Ivanova \& Tzeitlin (1987), mais ces gisements demeuraient inaccessibles aux spécialistes occidentaux.
A partir de 1990, l'ouverture des frontières politiques a favorisé l'accès d'équipes internationales à l'ensemble du domaine est-carpatique dans le cadre de projets de recherche multidisciplinaires. Dès lors, il fut possible d'élaborer une nouvelle séquence régionale basée sur les enregistrements de Molodova $\mathrm{V}$ en Ukraine occidentale, complétés par ceux de Mitoc-Malu Galben dans le nord-est de la Roumanie et de Cosautsi en République de Moldavie (Haesaerts et al. 2003). De même, le cadre chronologique de la séquence régionale du Danube moyen fut affiné grâce à un grand nombre de dates ${ }^{14} \mathrm{C}$ obtenues pour les principaux sites de Moravie (Svoboda 2001 ; Svoboda et al. 1994) et de Basse Autriche (Haesaerts 1990a ; Haesaerts et al. 1996 ; Neugebauer-Maresch 1999).

Les conditions nécessaires étaient donc réunies pour permettre l'élaboration d'une séquence chronostratigraphique et paléoclimatique globale à l'échelle de l'Europe centrale, intégrant un maximum de sites du Paléolithique supérieur. Pour ce faire, nous avons donné la préférence aux gisements pluristratifiés contenant des assemblages lithiques bien documentés, situés avec précision en stratigraphie. L'élaboration de la séquence globale implique par ailleurs la mise en place d'un schéma corrélatif associant les diffé- 
rents enregistrements régionaux selon le principe de la stratigraphie séquentielle. Ce principe prend en compte la distribution des principales unités lithostratigraphiques ainsi que les signatures paléoclimatiques des séquences considérées, lesquelles reposent sur une évaluation qualitative de l'environnement à partir des données pédosédimentaires et paléontologiques des enregistrements locaux (Haesaerts \& Van Vliet 1974). La démarche vise également à intégrer les différentes séries de dates radiométriques disponibles en considérant, en priorité, le positionnement stratigraphique et la qualité du matériel daté. On associe de la sorte deux ensembles de données établis indépendamment, dont la cohérence interne contrôle la validité du schéma corrélatif et fixe le cadre chronologique du système. Parmi le large éventail des dates ${ }^{14} \mathrm{C}$ reprises ci-dessous, un grand nombre de dates nouvelles furent obtenues à Groningen à partir de 1993 dans le cadre de projets financés par l'Etat belge et INTAS. Celles-ci furent réalisées essentiellement sur charbon de bois traité de manière à éliminer tout élément contaminant et à sélectionner du matériel bien identifié (Damblon et al. 1996 ; Damblon \& Haesaerts 2002).

\section{2 - LE BASSIN DU DANUBE MOYEN}

Ce domaine régional regroupe la Basse Autriche, la Moravie et la Slovaquie occidentale où sont concentrés les principaux gisements gravettiens (fig. 1). Ceux-ci sont répartis le long d'un axe joignant la vallée du Danube à la plaine baltique via la Moravie et la vallée du Váh. Les gisements de Willendorf et de Grubgraben sur le Danube, de Dolní Vestonice, de Stránská Skála et de Predmostí en Moravie jalonnent cet itinéraire qui aboutit à Spadzista dans le sud de la Pologne. Tous ces gisements sont associés à de longs enregistrements pédostratigraphiques bien documentés qui constituent l'ossature de la séquence régionale du pléniglaciaire moyen et du pléniglaciaire supérieur. D'autres gisements paléolithiques présentant des stratigraphies beaucoup plus réduites, mais néanmoins susceptibles d'être positionnées avec plus ou moins de précision dans la séquence régionale, ont également été sélectionnés. Citons en particulier KremsHundssteig, Albemdorf et Kamegg en Basse Autriche, Milovice et Petrkovice en Moravie, Nitra Cermán et Moravany-Lopata en Slovaquie. Par contre, les sites de Hongrie et de Slovaquie orientale, pour lesquels l'information stratigraphique est trop limitée ou auxquels nous n'avons pas eu accès, n'ont pas été considérés ici.

\section{1 - La seconde partie du pléniglaciaire moyen (de \pm 45000 à $26000 \mathrm{BP}$ )}

En Autriche-Moravie, l'essentiel de l'information stratigraphique et chronologique pour le pléniglaciaire moyen est enregistré le long de la vallée du Danube, à l'ouest de Vienne, principalement à Willendorf dans la Wachau et à Stratzing, ainsi qu'en Moravie, à Dolní Vestonice et à Stránská Skála (fig. 2). A Willendorf II et dans le site adjacent de Schwallenbach, cette période intègre deux unités distinctes préservées entre les couvertures loessiques pléniglaciaires, au sommet d'une basse terrasse du Danube (Haesaerts 1990b ; Haesaerts et al. 1996). La première (unité D) correspond à un dépôt colluvial et incorpore la couche culturelle 2 qui a fourni un premier assemblage lithique laminaire de type Paléolithique supérieur (Haesaerts \& Teyssandier 2003). La partie supérieure des colluvions, datée entre 41700 et $39900 \mathrm{BP}$, porte un sol brun carbonaté associé à une faune malacologique qui témoigne d'un épisode de réchauffement climatique majeur dénommé "Interstade de Willendorf" (Haesaerts et al. 1996). Par son faciès et sa position, cette pédogenèse peut être mise en parallèle avec le sol de Bohunice en Moravie associé à l'industrie lithique du Bohunicien et bien daté entre 43000 et 38750 BP (Valoch 1976 ; Svoboda et al. 1994 ; Haesaerts \& Teyssandier 2003).

A Willendorf et à Schwallenbach, la seconde unité qui couvre la période entre 40000 et $26000 \mathrm{BP}$ (unité C), traduit un environnement climatique nettement contrasté et relativement humide. Elle comprend un ensemble de loess sableux avec gleys de toundra qui alternent avec trois horizons humifères rapportés respectivement aux interstades de Schwallenbach I (39 000 à 37400 BP), de Schwallenbach II (vers $32000 \mathrm{BP}$ ) et de Schwallenbach III (vers $30500 \mathrm{BP}$ ) par référence au site du même nom où ces horizons sont préservés in situ (Haesaerts et al, 1996). Dans la coupe de Willendorf, la première couche aurignacienne (couche 3) datée 38880 et 37930 BP est incorporée à la base du sol Schwallenbach I; un hiatus sépare celui-ci des sols humifères Schwallenbach II et Schwallenbach III qui contiennent respectivement la couche aurignacienne 4 datée $32060 \mathrm{BP}$ et la couche gravettienne 5 datée 30500 BP. Ce doublet de sols humifères est probablement contemporain du sol de Stillfried B également présent à StratzingGalgenberg au nord de Krems où il est associé aux concentrations aurignaciennes datées entre \pm 31790 et \pm 29200 BP (Neugebauer-Maresch 1996)

En Moravie, la période 33000 - 30000 BP correspond globalement au sol W 2/3 décrit par B. Klíma (1963, 1995) à la base de la séquence loessique à Dolní Vestonice et à Pavlov (fig. 2). II s'agit également d'un horizon humifère complexe, le plus souvent étiré par solifluxion et localement dédoublé. Les charbons de bois qu'il contient ont été datés $29940 \mathrm{BP}$ dans la briqueterie et $31700 \mathrm{BP}$ dans la partie basse de la station A (Haesaerts 1985, 1990a), tandis que la partie inférieure de l'horizon a fourni un âge de 32850 BP à Dolní Vestonice I (Klíma 1995). Dans l'ensemble, le contexte archéologique du sol W 2/3 et des dépôts associés n'est guère documenté, excepté à Milovice où la couche culturelle aurignacienne datée 29230 BP est incorporée à des lentilles solifluées de sol humifère (Oliva 1989) et localement associée à des horizons cendreux datés $32030 \mathrm{BP}$ à la base et $28780 \mathrm{BP}$ au sommet (Oliva 2001). Enfin, à Stránská Skála, I'horizon humifère supérieur est également complexe et contient plusieurs concentrations aurignaciennes datées respectivement 32350 et 30980 BP (Svoboda et al. 1994), ce qui correspond à l'âge des sols Schwallenbach II et III à Willendorf. 
Dans la Wachau, mais aussi à Krems et à Stillfried, les dépôts limoneux sus-jacents aux sols Schwallenbach III et Stillfried B portent un épais gley de toundra témoin d'une péjoration climatique de peu antérieure à $26000 \mathrm{BP}$ qui précède directement les premiers apports éoliens du pléniglaciaire supérieur (fig. 2). Ces dépôts contiennent les témoins de plusieurs concentrations gravettiennes datées $28560 \mathrm{BP}$ à Willendorf et $27940 \mathrm{BP}$ à Krems-Hundssteig (Neugebauer-Maresch 2000), ainsi que la couche culture Ile principale d'Albemdorf attribuée à un Aurignacien évolué qui a fourni plusieurs dates sur os entre 26900 et 20 $500 \mathrm{BP}$ (Bachner et al. 1996), mais fut datée récemment de 28360 BP sur charbon de bois (Haesaerts et al. 2004). Enfin, une seconde génération d'occupations gravettiennes est associée à la partie sommitale du gley de toundra ; c'est le cas de la couche culturelle 6 de Willendorf datée 26500 et 26150 BP et probablement aussi de l'occupation principale d'Aggsbach qui a fourni des âges équivalents (Otte 1981 ; Haesaerts 1985, 1990a).

Dans la plupart des secteurs de Dolní Vestonice et de Pavlov, les dépôts directement sus-jacents au sol W 2/3 atteignent en moyenne un mètre d'épaisseur et se composent d'une succession de lentilles limoneuses et de couches loessiques solifluées ; ces dépôts sont affectés par une importante pédogenèse de type gley de toundra (gley G1, cf. Klíma 1963, 1969), équivalente à celle développée au sommet de la séquence du pléniglaciaire moyen à Wllendorf, à Krems et à Stillfried $\mathrm{B}$ (fig. 2). C'est dans la moitié supérieure de ce complexe que se situe la majorité des couches culturelles du Pavlovien dans les secteurs Dolní Vestonice I, Dolní Vestonice II et Pavlov I, pour lesquelles on dispose d'un grand nombre de dates radiométriques cohérentes comprises entre 27500 et \pm 25500 BP (fig. 2 et 3 ; Klíma 1995 ; Svoboda et al. 1994 ; Svoboda 2001). Par ailleurs, dans les profils du secteur Dolní Vestonice II, les occupations attribuées au Pavlovien sont distinctement postérieures à un léger horizon humifère fortement étiré, daté vers $28000 \mathrm{BP}$, qui paraît bien traduire un épisode climatique positif également enregistré par la palynologie (Svobodová 1991), dénommé épisode interstadiaire de "Dolní Vestonice» (Haesaerts 1990a ; Haesaerts et al. 1996).

\section{2 - Le pléniglaciaire supérieur et le Tardiglaciaire (de 26000 à $10000 \mathrm{BP}$ )}

En Autriche, cette longue période a connu plusieurs phases de sédimentation loessique dont la stratigraphie fut établie à partir des enregistrements complémentaires de Willendorf, Krems-Hundssteig, Stillfried et Grubgraben (fig. 2). A Willendorf, la première phase loessique correspond à l'unité $B$ datée entre \pm 26000 et $\pm 25000 \mathrm{BP}$; elle comprend deux couches de loess poudreux séparées par un sol humifère incipient qui contient la couche culturelle gravettienne 8 datée entre 25800 et 25230 BP dans la coupe du champ de fouilles ouverte en 1981 et 1993 (Haesaerts et al. 1996). La couche 9 à Gravettien évolué (Willendorfien cf. Kozlowski 1986), située dans le loess supérieur environ un mètre au-dessus de la couche 8 , n'a pas été rencontrée dans la coupe de 1993 mais fut datée 24910 BP sur la partie centrale d'un gros os provenant des fouilles de J. Bayer (1930). Notons à ce propos que la position stratigraphique de la célèbre vénus de Willendorf, classiquement rapportée à la couche 9, est contestée par cet auteur, lequel attribue cette vénus à une petite concentration lithique nettement distincte, située environ $50 \mathrm{~cm}$ en dessous de la couche 9 (Bayer 1930 : 54).

La partie médiane de la couverture loessique, dépourvue de repères chronologiques, est accessible en position de versant dans plusieurs sites de Basse Autriche. A Schwallenbach, à Krems-Hundssteig et à Stillfried $B$, elle se compose d'un complexe de loess poudreux avec plusieurs petits gleys de toundra, surmonté d'un dépôt loessique finement lité. Cette succession est également présente dans la partie inférieure de la séquence de Grubgraben, dans une large combe ouverte vers le sud en direction du Danube, sous un épais

Figure 2 - Principales séquences pour la Basse Autriche, la Moravie, la Slovaquie occidentale et le sud de la Pologne : stratigraphie, archéologie et dates ${ }^{14} \mathrm{C}$.

Symboles graphiques des Figures 2 à 8.1 : loess ; 2 : limons ; 3 : sable limoneux ; 4 : sable ; 5 : craie ; 6 : graviers ; 7 : calcaire ; 8 : horizon illuvié (B2t) ; 9 : horizon humifère fortement développé ; 10 : horizon humifère faiblement développé ; 11 : horizon brun-jaune bioturbé ; 12 : krotovines ; 13 : horizon déferrifié (gley de toundra) ; 14 : hydroxydes de fer ; 15 : coin de glace ; 16 : fente de gel ; 17 : Moustérien ; 18 : industries de transition ; 19 : Aurignacien ; 20 : Gravettien ancien et moyen, Pavlovien inclu ; 21 : Gravettien supérieur à pointes à cran ; 22 : Epigravettien et faciès associés ; 23 : Paléolithique supérieur indéterminé ; 24 : Magdalénien. Abréviations (figure 2). Schwal : Schwallenbach ; St. B : Stillfried B ; DV : Dolní Vestonice ;

Pavl : Pavlov : DV br : Dolní Vestonice briqueterie.

Figure 2 - Main sequences from Lower Austria, Moravia, Western Slovakia and South Poland: stratigraphy, archaeology and radiocarbon dates.

Graphic symbols for Figures 2 to 8. 1: loess; 2: loam; 3: silty sand; 4: sand; 5: chalk; 6: gravel; 7: limestone; 8: illuviated horizon (B2t); 9: strong humiferous horizon; 10: weak humiferous horizon; 11: yellowish brown bioturbated horizon; 12: krotovinas; 13: bleached horizon (tundra gley): 14: iron staining; 15: ice wedge cast; 16: frost wedge; 17: Mousterian; 18: transitional industries; 19: Aurignacian; 20: Early and Middle Gravettian, including Pavlovian; 21: Late Gravettian with shouldered points; $22:$ Epigravettian and others; 23: poorly documented Upper Palaeolithic; 24: Magdalenian.

Abbreviations (Figure 2). Schwal: Schwallenbach; St. B: Stillfried B; DV: Dolní Vestonice; Pavl: Pavlov: DV br: Dolní Vestonice brickyard. 


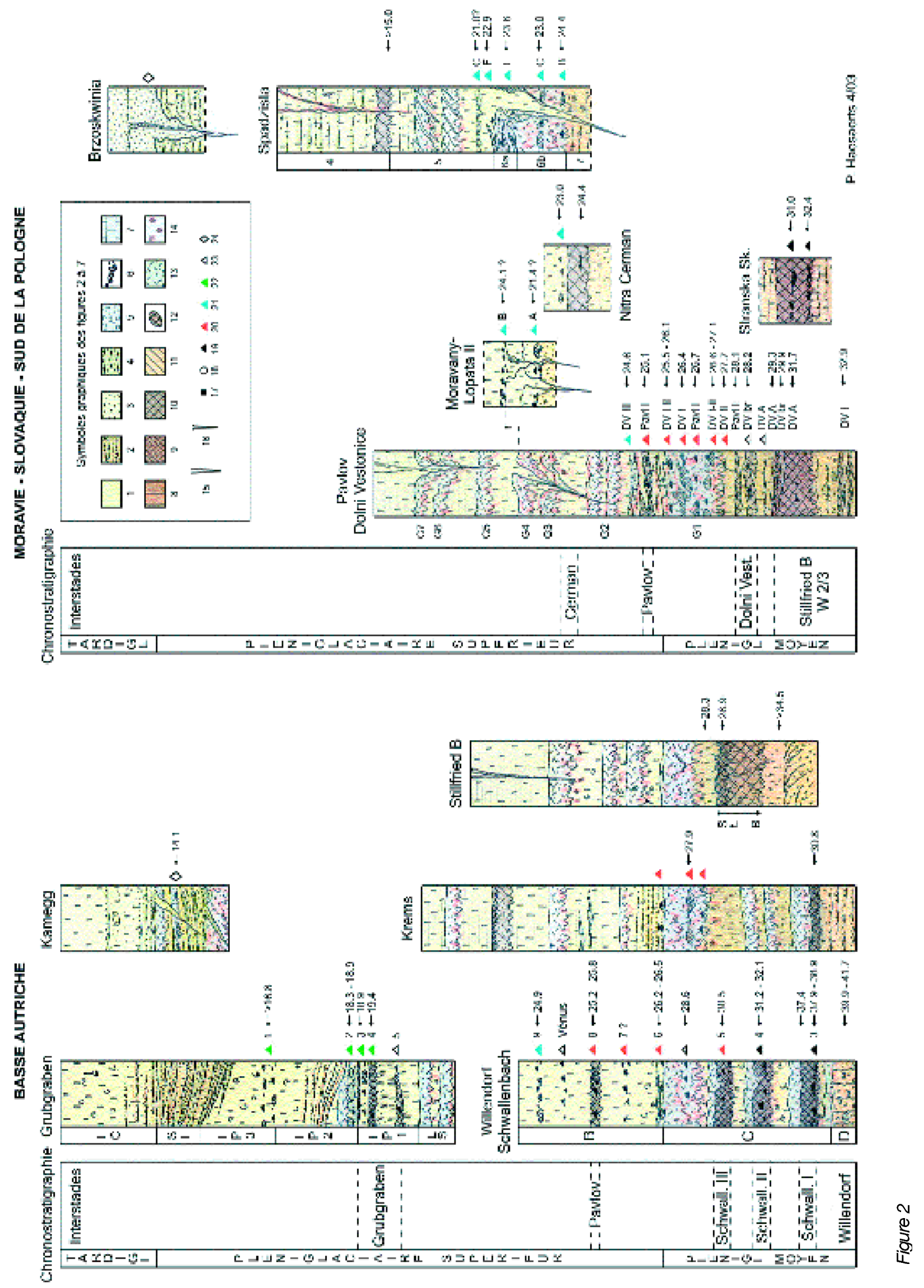


complexe de trois dépôts loessiques séparés par des chenaux sableux (fig. 2). En particulier, la présence dans la partie inférieure du premier dépôt loessique de trois horizons humifères associés à plusieurs couches culturelles «épigravettiennes" (Montet-White 1990) datées entre 19380 et $18380 \mathrm{BP}$, constitue un repère précieux rapporté à la phase climatique de "Grubgraben» (Haesaerts 1990c ; Haesaerts et al. 2004). Le caractère interstadiaire de ces courts épisodes de pédogenèse est bien exprimé au niveau de l'horizon humifère contenant la couche culturelle 4 que l'on peut suivre sur une cinquantaine de mètres de distance le long du chemin creux qui jouxte le gisement. L'ensemble atteste donc d'une sédimentation complexe dans un contexte climatique contrasté et relativement humide au cours de la seconde partie du pléniglaciaire supérieur, sous végétation à dominante steppique avec présence du pin cembra au voisinage du site lors des courts épisodes interstadiaires (Haesaerts 1990c : 30). A Grubgraben, la sédimentation s'est probablement poursuivie jusqu'au Tardiglaciaire, ce dont témoigne le caractère nettement plus sec du contenu malacologique de l'unité loessique supérieure (Haesaerts 1990c). Cette dernière génération loessique est également présente à Kamegg dans le vallée de la Kamp au-dessus de dépôts sablo-limoneux à fentes de gel qui contiennent une industrie rapportée au Magdalénien (Otte 1981) datée récemment sur os à Groningen de 14100 BP (Haesaerts et al. 2004).

En Moravie, c'est la couverture loessique de Dolní Vestonice, au pied des Monts Pavlov, qui sert de référence pour le pléniglaciaire supérieur (Demek \& Kukla 1969 ; Klíma 1963, 1969, 1995). Parmi les gleys de toundra G2 à
G7, qui constituent la signature de cette formation, I'horizon G2 et le doublet G3-G4 associé à un réseau de coins de glace sont les mieux exprimés, un second réseau de coins de glace étant présent au sommet du doublet G6-G7 (fig. 2). A Pavlov I, à Dolní Vestonice II mais aussi dans le secteur G de Milovice (Oliva 1989), un petit groupe de dates proches de $25000 \mathrm{BP}$ obtenues à Groningen sur charbon de bois pourrait témoigner de la persistance des occupations du Gravettien moyen au cours de la phase initiale du pléniglaciaire supérieur, comme c'est le cas des couches 7 et 8 à Willendorf, mais ici les processus de solifluxion qui affectent le sommet du gley G1 ne permettent pas de les différencier stratigraphiquement des occupations antérieures. Toutefois, dans les profils du chemin creux à l'est de la station Pavlov II, B. Klíma signale la présence d'un horizon humifère décimétrique contenant des éléments de la couche culturelle, au contact du gley G1 et de la couverture des loess sus-jacents (Klíma 1976) ; ce petit horizon humifère, probablement équivalent à celui de la couche culturelle 8 de Willendorf, traduirait également une légère amélioration climatique voisine de $25500 \mathrm{BP}$ dénommée oscillation de «Pavlov» (Haesaerts 1990a).

Quelques témoins d'occupations plus tardives existent cependant dans d'autres stations de la région, notamment à Dolní Vestonice III où une concentration lithique proche du Gravettien supérieur, datée 24560 BP, est présente dans la partie inférieure des loess (Skrdla et al. 1996 ; Svoboda 2001). C'est le cas également de la concentration d'ossements de mammouth du secteur C-D à Milovice qui a fourni un âge de 22250 BP et appartient à la partie médiane de la couverture loessique sans plus de

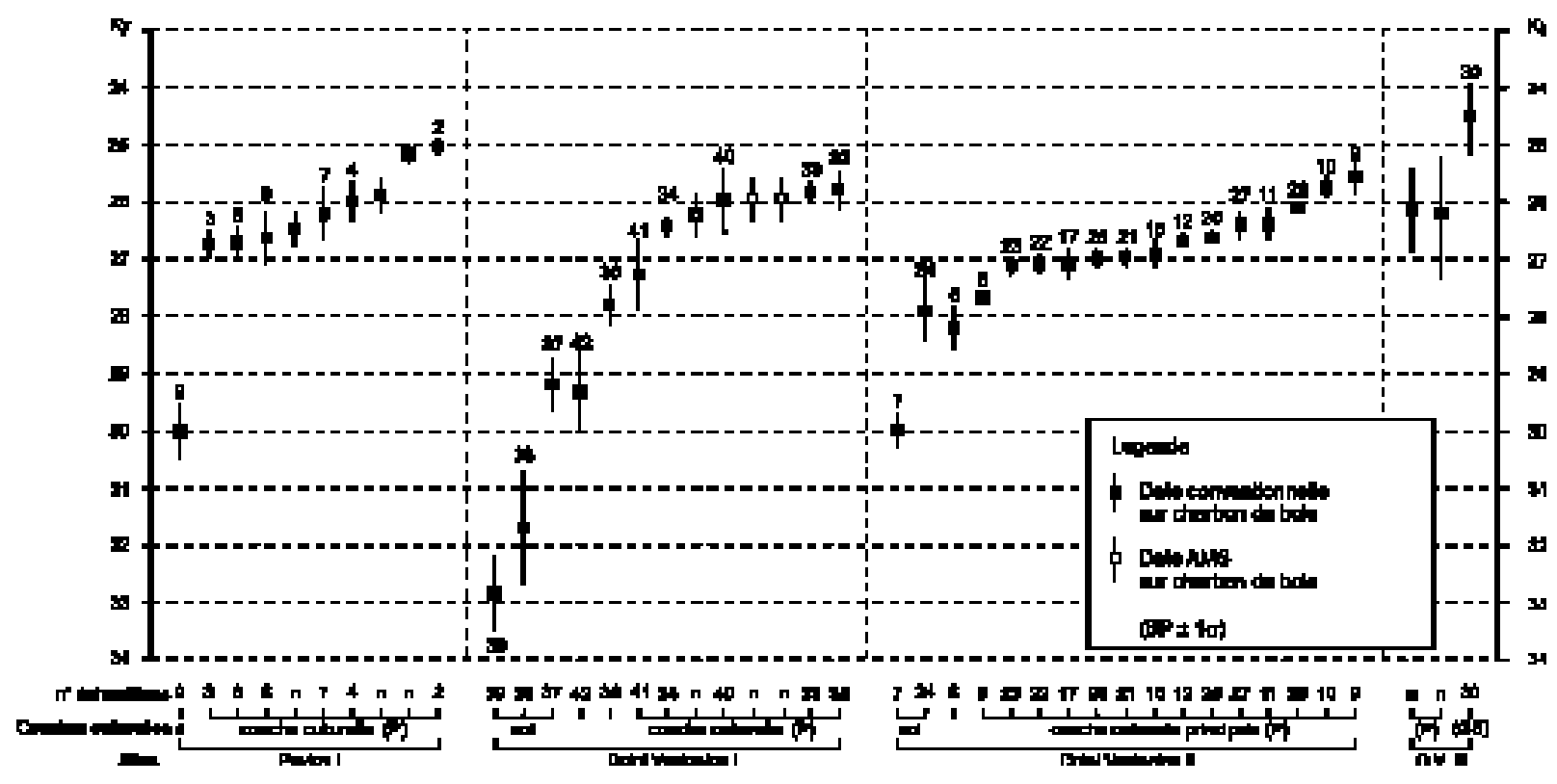

Figure 3 - Pavlov et Dolní Vestonice (Moravie) : distribution des dates ${ }^{14} \mathrm{C}$ (d'après Damblon et al. 1996).

Figure 3 - Pavlov and Dolní Vestonice (Moravia): distribution of the radiocarbon dates (after Damblon et al. 1996). 


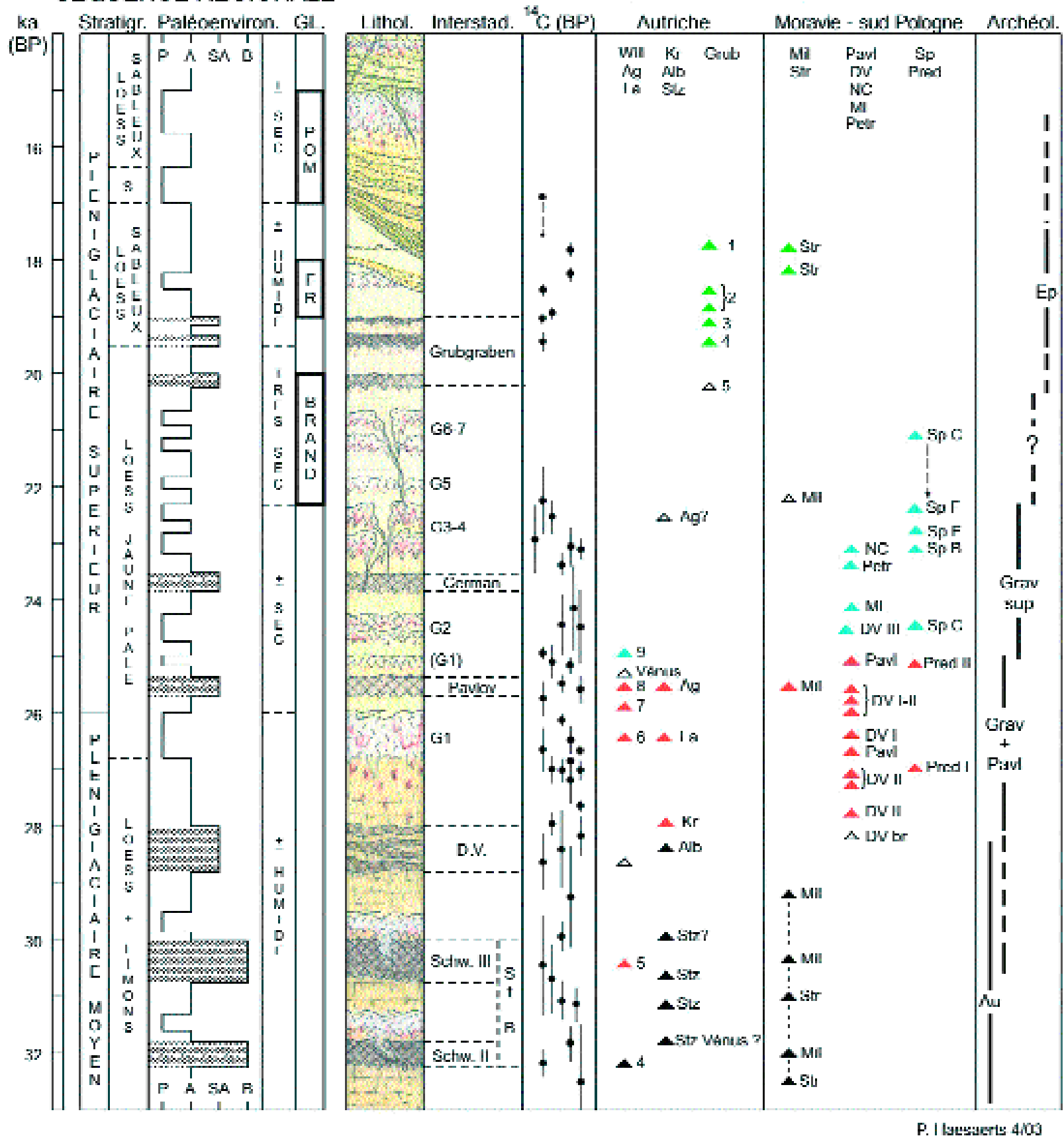

Figure 4 - Séquence régionale pour la Basse Autriche, la Moravie, la Slovaquie occidentale et le sud de la Pologne (symboles graphiques : voir figure 2).

Abréviations. $S$ : sable; Paléoenviron : paléoenvironnement; $P$ : périglaciaire avec gel profond ou permafrost actif; $A$ : arctique; $S A$ : subarctique; $B$ : boréal; Interst : interstades; GL : stades glaciaires; POM : Poméranie; FR : Frankfurt;

BRAND : Brandenburg; Interstad : interstades; DV : Dolní Vestonice; Schw : Schwallenbach; St B : Stillfried B; Will : Willendorf; Kr : Krems-Hundssteig; Alb : Alberndorf; Stz : Stratzing-Galgenberg; Gro : Grossweikersdorf; Grub : Grubgraben;

Ag : Aggsbach; La : Langenlois; Str : Stránská Skála ; Mil : Milovice; Pavl : Pavlov; NC : Nitra Cermán; ML : Moravany-Lopata; Sp : Spadzista; Petr : Petrkovice; Pred : Predmosti; Ep : Epigravettien et faciès associés;

Grav sup : Gravettien supérieur à pointes à cran; Grav + Pavl : Gravettien et Pavlovien; Au : Aurignacien.

Figure 4 - Regional sequence for Lower Austria, Moravia, Western Slovakia and South Poland (graphic symbols as in fig. 2). 
précision (Oliva 1989). Quant aux dates 18400 et 15350 $\mathrm{BP}$ réalisées sur la fraction humifère des loess de la briqueterie à Dolní Vestonice (Demek \& Kukla 1969 ; Klíma 1995), elles sont dépourvues de signification chronologique car fortement rajeunies comme le sont toutes les dates sur humus obtenues pour les loess d'Europe centrale (Haesaerts 1985, 1990a).

Dans ce contexte, les enregistrements de Spadzista près de Cracovie (Escutenaire et al. 1999) et de Nitra Cermán (Bárta 1980) en Slovaquie occidentale (fig. 2) permettent de préciser quelque peu le cadre chronologique et archéologique de la couverture loessique pléniglaciaire de Moravie. A Spadzista, l'épais gley de toundra et les dépôts soliflués sus-jacents associés à un réseau de grands coins de glace (sous-unités $6 \mathrm{~b}$ et $6 \mathrm{a}$ ), qui contiennent les principales occupations gravettiennes à pointes à cran datées entre 24380 et 23040 BP sur charbon de bois, seraient équivalents au doublet G3-G4 de Dolní Vestonice. De même, l'horizon humifère de peu postérieur à $24440 \mathrm{BP}$, présent à Nitra Cermán sous la couche gravettienne à pointes à cran datée $23000 \mathrm{BP}$ sur charbon de bois à la base de la couverture loessique (Bárta 1980), serait à rapporter à un second épisode interstadiaire interne au pléniglaciaire supérieur nettement distinct de l'oscillation de Pavlov, épisode que nous désignons ici sous le nom de oscillation de «Cermán» (fig. 2 et 4).

Par ailleurs, à Spadzista, le loess lité avec gleys de toundra peu développés (unité 5) postérieur à la péjoration climatique de l'unité 6 , contient les derniers ateliers gravettiens datés 21000 BP sur os. D'après Kozlowski (1996, 1998) et Escutenaire et al. (1999), cette unité loessique, également bien exprimée dans les sites paléolithiques du nord de la Moravie et de Slovaquie occidentale où elle recouvre les concentrations du Gravettien à pointes à cran, se serait mise en place entre \pm 21000 et $\pm 17000 \mathrm{BP}$, soit pendant et juste après le stade glaciaire de BrandenburgLezsno. Cette fourchette chronologique nécessite cependant quelques réserves car elle repose sur un corpus de données hybrides associant des âges radiocarbone sur os provenant de laboratoires différents, ainsi que des âges TL à larges erreurs statistiques (Escutenaire et al. $1999: 23$ ). En particulier, la date sur os de la couche 5 à Spadzista constitue probablement un âge minimum, comme c'est souvent le cas pour du matériel osseux préservé dans les loess carbonatés (Damblon et al. 1996 ; Haesaerts et al. 2003). Dès lors, un âge plus proche de 23000 BP pour la base de la couverture loessique supérieure et pour la fin des occupations gravettiennes à pointes à cran nous paraît plus probable. Le même phénomène pourrait expliquer la discordance chronologique entre les dates sur os des couches A et B à Moravany-Lopata (Pazdur 1998), la date de $21400 \mathrm{BP}$ issue de la couche inférieure (A) étant probablement rajeunie et moins fiable que la date de 24100 BP obtenue pour la couche supérieure (B).

Enfin, dans le sud de la Pologne, la seconde moitié du pléniglaciaire supérieur se caractérise aussi par une augmentation de la composante sableuse des dépôts de couverture et se termine également par une dernière péjoration clima- tique. Celle-ci se marque par un réseau de grands coins de glace développé au sommet des loess sableux à Brzoskwinia (Sobczyk 1995), lequel semble s'être maintenu pendant la mise en place des sables de couverture associés à l'occupation du site par les chasseurs du Magdalénien au cours de la première moitié du Tardiglaciaire.

\section{3 - LE DOMAINE EST-CARPATIQUE}

Cette vaste région située à l'est des contreforts des Carpates, drainée par le Prut et le Dniestr, possède un potentiel de gisements paléolithiques exceptionnel prospecté dès 1929 par N. Morosan (1938). Parmi les nombreux sites de plein air connus en Ukraine occidentale, en République de Moldavie et en Roumanie, et qui demeurent accessibles, seuls quelques-uns présentent de longues séquences stratigraphiques associées à des occupations pluristratifiées du Paléolithique supérieur. Molodova $\mathrm{V}$ sur la rive ukrainienne du Dniestr fut exploité par A. Chemysh (1959, 1987) et I. Ivanova (Ivanova \& Tzeitlin 1987) ; Cosautsi sur la rive moldave du Dniestr et Mitoc-Malu Galben sur la rive roumaine du Prut furent fouillés au cours des années quatre-vingt (Borziac 1991, 1993 ; Chirica 1989, 2001).

Au cours de la demière décennie, ces sites ont fait l'objet d'études pluridisciplinaires dans le cadre de programmes de recherche internationaux et constituent la structure de base de la séquence régionale (Otte et al. 1996 ; Haesaerts et al. 2003 ; Noiret 2004). Ils représentent trois enregistrements pédosédimentaires et paléoclimatiques complémentaires avec de multiples couches du Paléolithique supérieur, encadrés par une chronologie ${ }^{14} \mathrm{C}$ solide et bien documentée pour la période $33000-10000$ BP. Dans ce système, la résolution optimale de chaque séquence fut contrôlée par la dynamique sédimentaire et par la position du site dans le paysage (fig. 5 et 6).

Cosautsi, au sommet de la première terrasse du Dniestr, a foumi une séquence de haute résolution pour la seconde moitié du pléniglaciaire supérieur et le Tardiglaciaire, tandis que Mitoc-Malu Galben, sur le versant de la deuxième terrasse du Prut, a favorisé un enregistrement complémentaire au cours de la phase finale du pléniglaciaire moyen et de la première partie du pléniglaciaire supérieur. Par ailleurs, Molodova V, dans la continuation de la seconde terrasse du Dniestr située environ 20 mètres au-dessus du talweg, a conduit à la préservation d'une longue séquence couvrant la majeure partie du Pléistocène supérieur (Ivanova \& Tzeitlin 1987) avec une haute résolution chronologique pour la période 33000 - 10000 BP (Haesaerts et al. 2003).

\section{1 - Partie supérieure du pléniglaciaire moyen (de \pm 33000 à $26000 \mathrm{BP}$ )}

A Mitoc-Malu Galben, cette période correspond aux unités 13 à 7 dont le grand développement est lié à la position du site au pied du talus de la seconde terrasse du Prut (fig. 5 et 6). Cette accumulation loessique repré sente un enregistrement cyclique semi-continu de six 

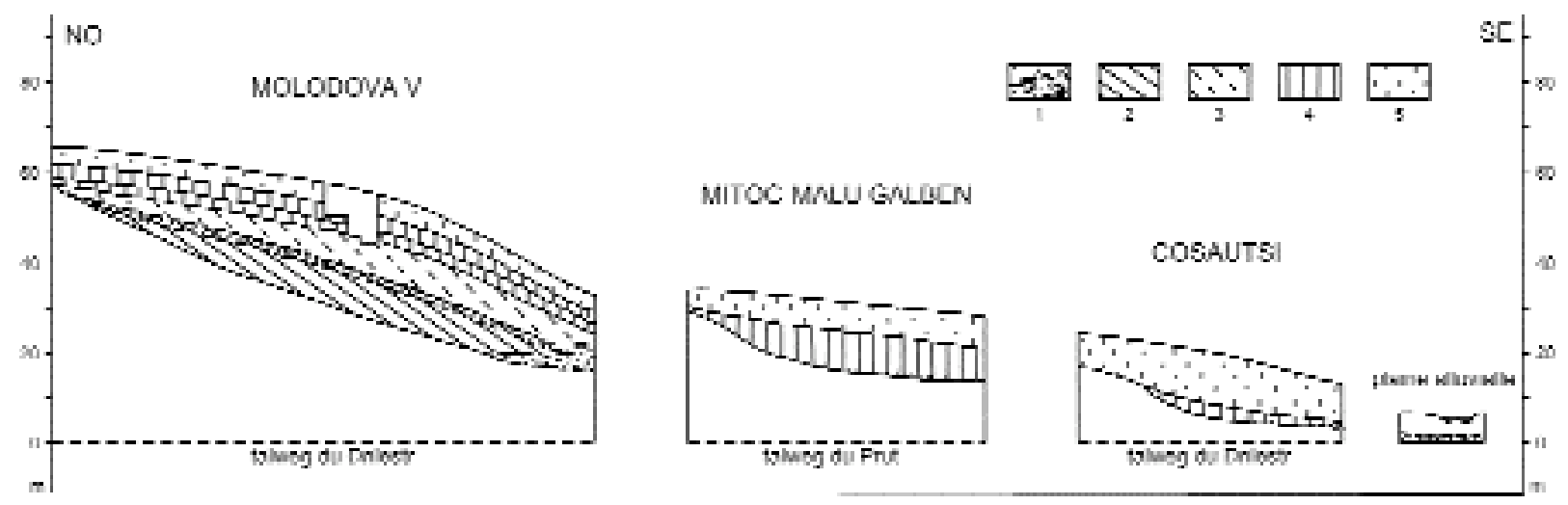

Figure 5 - Contexte géomorphologique des sites de Molodova V, Mitoc-Malu Galben et Cosautsi.

Symboles graphiques. 1 : graviers fluviatiles; 2 : dépôts du début glaciaire (Molodova $V$ : unités 1 à 4); 3 : limons du pléniglaciaireinférieur (Molodova V : unité 5); 4 : loess et paléosols du pléniglaciaire moyen (Molodova V : unités 6 à 10; Mitoc : unités 13 à 7); 5 : loess du pléniglaciaire supérieure et du Tardiglaciaire.

Figure 5 - Geomorphological background of Molodova V, Mitoc-Malu Galben and Cosautsi.

Graphic symbols; 1 : fluviatile gravel; 2 early glacial deposits (Molodova V : units 1 to 4); 3 : early pleniglacial loam

(Molodova V : unit 5); 4 : middle pleniglacial loess-palaeosols (Molodova V : units 6 to 10; Mitoc : units 13 to 7 ); 5 : late pleniglacial and late glacial loess.

horizons humifères d'intensité décroissante, avec une sédimentation colluviale (unités 13 à 11) suivie par des apports de limons loessiques (unités 10 à 7). La distribution des dates ${ }^{14} \mathrm{C}$ dans cet ensemble d'unités a permis de situer ces six épisodes interstadiaires nommés Malu Galben 13 à 8, respectivement vers 33000 BP (Malu Galben 13), 31200 BP (Malu Galben 12), 30500 BP (Malu Galben 11), entre \pm 29000 et 27700 BP (Malu Galben 10 et Malu Galben 9) et vers 27000 BP (Malu Galben 8). Les unités 12 à 8 contiennent de nombreux ateliers aurignaciens, tandis que l'unité 7 incorpore les p remiers ateliers gravettiens (Otte et al. 1996 ; Noiret 2004) et porte un épais gley de toundra daté autour de 26000 BP, indicateur d'un refroidissement drastique qui conclut le pléniglaciaire moyen et constitue un premier m a rqueur stratigraphique (fig. 6).

A Molodova V, la période de 33000 à $26000 \mathrm{BP}$ correspond au pédocomplexe supérieur (unité 10) développé dans les colluvions de la partie sommitale de la séquence du pléniglaciaire moyen (fig. 6 et 7). Ce pédocomplexe comprend deux sols bruns de type para-rendzine (sous-unités 10-1 et 10-2) datés autour de 32600 et de 30400 BP et donc contemporains des épisodes Malu Galben 13 et Malu Galben 10. Ces sols sont suivis par un horizon humifère gris foncé (sous-unité 10-3) daté entre 29650 et 27700 BP, qui correspond aux épisodes Malu Galben 10 et Malu Galben 9 ; au-dessus de cet horizon existe également un épais gley de toundra bien daté entre 26640 et 25760 BP. A la différence de Mitoc-Malu Galben, le contenu archéologique de l'unité 10 à Molodova $V$ est limité aux couches gravettiennes 10 et 9 qui appartiennent distinctement à l'épisode froid séparant les sols $10-2$ et 10-3 (fig. 6).

\section{2 - Première partie du pléniglaciaire supérieur (de 26000 à \pm 20000 BP)}

A Molodova et à Mitoc, cette période enregistre à nouveau une sédimentation cyclique avec deux ensembles de loess sableux jaune pâle qui reflètent des conditions climatiques froides et encore contrastées. Le premier ensemble de loess correspond aux unités 11 et 12 de Molodova et aux unités 6 à 4 de Mitoc. II comprend trois apports éoliens altemant avec des gleys de toundra ; le gley supérieur daté autour de 23000 BP (unité C) est le mieux développé et constitue un second marqueur stratigraphique. Ce premier complexe loessique présente encore deux horizons humifères associés aux épisodes climatiques interstadiaires Malu Galben 6 et Malu Galben 4 (fig. 6 et 7), datés respectivement vers $25500 \mathrm{BP}$ à Molodova (sous-unité 11-2) et entre 23830 et 23290 BP à Mitoc (unité 4).

Dans ces deux sites, le premier complexe loessique contient les couches culturelles gravettiennes principales. A Mitoc, les concentrations gravettiennes II et III, datées entre 26450 et 24780 BP, appartiennent au Gravettien moyen tandis que la partie supérieure de la concentration III et la concentration IV datées entre 24780 et 23290 BP sont rapportées au Gravettien supérieur à pointes à cran (Otte et al. 1996 ; Noiret 2004). A Molodova $\mathrm{V}$, la situation est légèrement différente car seule la couche culturelle 8 dans l'horizon humifère 112 appartient au Gravettien moyen. Quant à la couche culturelle 7 , rapportée au Gravettien à pointes à cran (Otte 1981; Kozlowski 1986), elle fut datée entre 25280 et 25130 BP dans la partie supérieure du loess 11-3; la couche 7 fut également rencontrée par $A$. Chernysh 
(1987) dans I'horizon humifère 12-1 daté 23650 BP et dans le gley de toundra sus-jacent (sous-unité 12-2) daté 23000 BP (Haesaerts et al. 2003).

En ce qui concerne le second complexe loessique du pléniglaciaire supérieur, les unités 3 et 2 de Mitoc et l'unité 13 à Molodova $\mathrm{V}$ reflètent clairement des conditions environnementales devenant de plus en plus sèches entre \pm 23000 et \pm 20000 BP (Haesaerts et al. 2003). Au cours de cette période, les apports loessiques alternent avec trois épisodes de gel profond marqués par des gleys de toundra et un court épisode positif (Molodova 13-2) marqué par un horizon bioturbé daté 21540 BP. Quant aux témoins archéologiques, ils se limitent dans les deux sites à de petites concentrations atypiques dispersées à différents niveaux dans le loess.

\section{3 - Seconde partie du pléniglaciaire supérieur (de \pm 20000 à \pm 14500 BP)}

Dans le domaine est-carpatique, cette période se caractérise également par une évolution climatique cyclique. Celle-ci est la mieux enregistrée à Cosautsi sur la basse terrasse du Dniestr (cycles VII à IV) ainsi que dans la partie supérieure de Molodova V (unité 14).

A Cosautsi, la période comprise entre \pm 20000 et \pm 17200 BP correspond aux cycles VII à IV (pro-parte) ; elle se marque par une diminution des apports loessiques et par la formation d'une série de six horizons humifères d'intensité décroissante, dans un contexte climatique relativement humide avec persistance de parcelles boisées associant conifères et décidus le long de la vallée du Dniestr (Medianik 1994 ; Haesaerts et al. 2003). L'horizon humifère de l'unité 7-2 associé à l'épisode interstadiaire Cosautsi VII-2 est de peu antérieur à $20000 \mathrm{BP}$ (Haesaerts et al. 2003 : 179), tandis que les horizons des unités 6-4, 6-2, 5-4, 5-2, 4-4 et 43 , associés aux épisodes climatiques Cosautsi VI-4 à IV-3, sont bien datés entre 19400 et $17130 \mathrm{BP}$ par une double série de dates cohérentes obtenues sur charbon de bois à Groningen et sur os à Oxford (Haesaerts et al. 1998 ; Otte et al. 1996). Cette séquence enregistre également plusieurs épisodes de gel profond, en particulier vers 20000 BP (unité 7-1), $18300 \mathrm{BP}$ (unité 6-1) et $17200 \mathrm{BP}$ (unité 4-4).

Dans le domaine est-carpatique, les dépôts de cette période ont fourni de nombreuses concentrations épigravettiennes (Noiret 2004). A Cosautsi, pas moins de 17 couches culturelles distinctes ont été rencontrées depuis l'unité 7-1 jusqu'au sommet de l'unité 5-1 (Borziac 1991, 1993). A Molodova V, la première occupation épigravettienne datée 20400 BP (couche culturelle 6) appartient à l'épisode interstadiaire Molodova 14-1 équivalent à Cosautsi VII, tandis que les couches 5 et 4 situées entre \pm 19000 et 17800 BP pourraient correspondre aux épisodes Cosautsi VI-2 et Cosautsi V-4 (fig. 6 et 7).

Finalement, à partir de \pm 17200 , une modification drastique du climat vers des conditions extrêmes avec plusieurs épisodes à permafrost est enregistrée à Cosautsi (cycle IV) et dans la partie supérieure de la séquence de Mitoc (sous-unité 1b). Ces conditions se traduisent par une activité éolienne intense avec des apports croissants de sables locaux et des processus de fonte. Cette phase climatique se termine par un épisode à permafrost marqué par un dernier gley de toundra bien développé (unité 4-1 à Cosautsi et probablement sous-unité 14-3 à Molodova V) de peu postérieur à 16000 BP. Seules de petites concentrations préservées dans les sédiments sableux du cycle IV à Cosautsi témoignent d'activités humaines au cours de cette période du pléniglaciaire supérieur terminal.

\section{4 - Le Tardiglaciaire (de \pm 14500 à 10000 BP)}

A partir de $\pm 14500 \mathrm{BP}$, une période de sédimentation éolienne sous des conditions climatiques plutôt sèches a favorisé le dépôt d'une dernière couverture loessique sur les versants des vallées du Prut et du Dniestr. Les apports éoliens se sont poursuivis, semble-t-il, jusqu'à la fin du Dryas avec des interruptions temporaires accompagnées de la formation de sols humifères à proximité et dans les fonds de vallée au cours du Bölling et de l'Alleröd (Cosautsi III et Cosautsi II). A Molodova V, les dates comprises entre 13370 et $10940 \mathrm{BP}$ obtenues par I. Ivanova pour les couches culturelles 3 à 1 de l'unité loessique 14-4, suggèrent un âge tardiglaciaire pour ce loess et les industries qu'il contient. Cette interprétation est toutefois contestée par D. Nuzhnyi (comm. pers.) sur la base d'une étude comparative des assemblages lithiques de ces deux sites, laquelle plaiderait en faveur d'un âge plus ancien, voisin de $17500 \mathrm{BP}$ pour les couches 3 à 1 de Molodova.

\section{4 - LA SÉQUENCE STRATIGRAPHIQUE GLOBALE}

La mise en parallèle des séquences régionales établies de part et d'autre des Carpates constitue la seconde étape de notre démarche ; basée sur l'analyse séquentielle des ensembles pédosédimentaires et de leurs signatures climatiques, celle-ci vise en particulier une intégration des données paléoclimatiques et archéologiques à l'échelle de l'Europe centrale pour la période comprise entre 33000 et 10000 BP. II importe de souligner ici le caractère exceptionnel de la séquence climatique du domaine est-carpatique, dont la chronologie repose sur de longues séries de dates radiométriques réalisées pour l'essentiel sur charbon de bois de conifères. Rappelons à ce propos que les enregistrements pédosédimentaires et les dates radiocarbone constituent des bases de données indépendantes mais complémentaires, qui par leur cohérence interne renforcent et fixent la chronologie du système. La limite inférieure de notre schéma fut donc située vers $33000 \mathrm{BP}$ car au-delà, les données chronologiques précises font défaut dans le domaine est-carpatique, notamment à Molodova V (fig. 7), tandis que dans le domaine occidental, les stratigraphies de la période antérieure à 33000 BP sont trop fragmentaires.

La principale caractéristique du schéma corrélatif établi de la sorte pour le domaine loessique d'Europe centrale réside dans la remarquable reproductibilité de l'ensemble des événements de part et d'autre des Carpates ; cela concerne non seulement les grandes unités lithostratigraphiques 


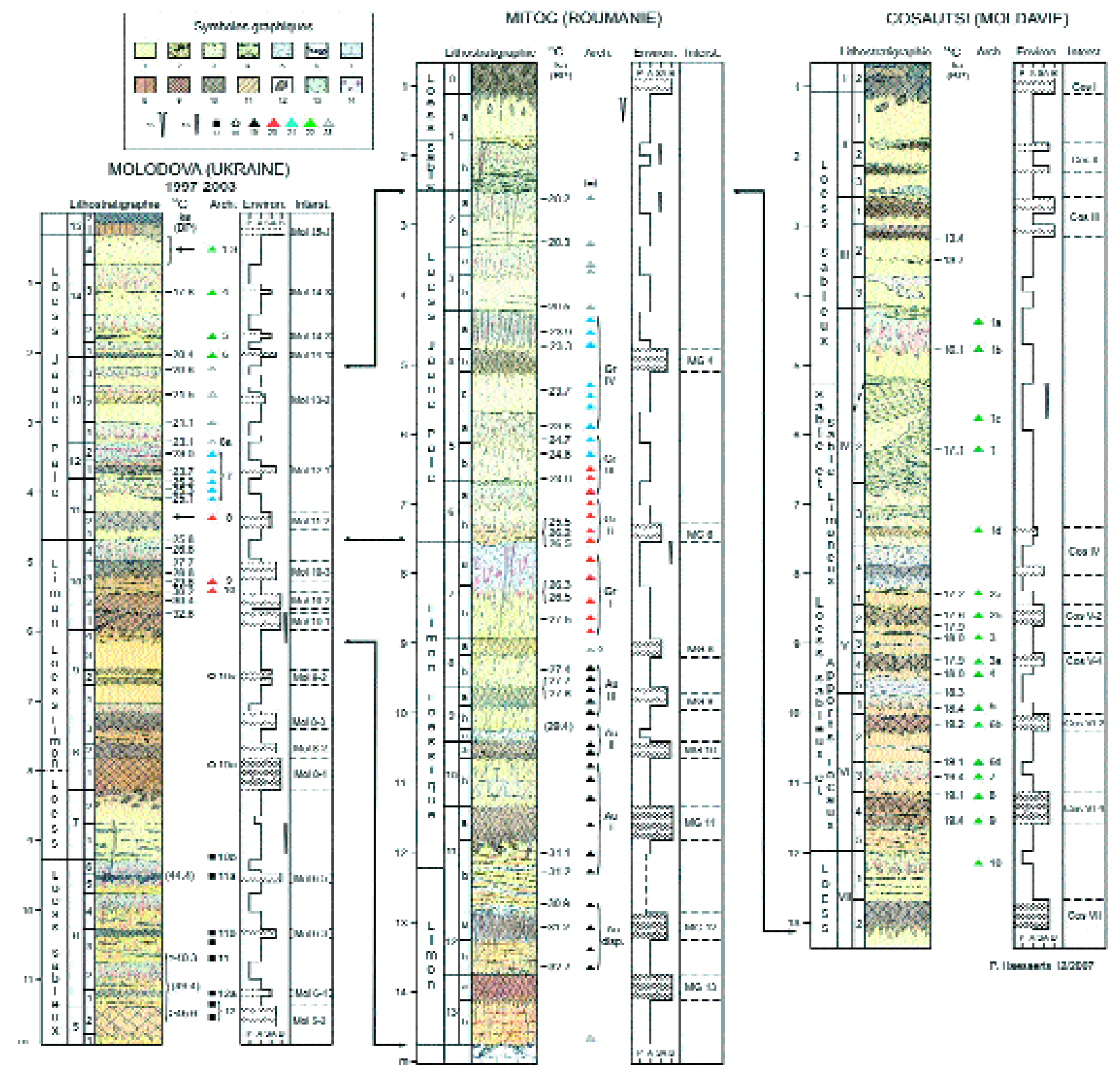

Figure 6 - Principales séquences du domaine est-carpatique (symboles graphiques : voir fig. 2).

Abréviations. H : Holocène; TGL : Tardiglaciaire; $L$ : loess; $L S$ : loess sableux; Lm : limons; SL : sable limoneux; $S$ : sable; hum : horizons humifères; gl $t$ : gley de toundra; MG : Malu Galben; Mol : Molodova V; Cos : Cosautsi; Arch : archéologie; Paléoenviron : paléoenvironnement; $P$ : périglaciaire avec gel profond ou permafrost actif; $A$ : arctique; SA; subarctique; $B$ : boréal.

Figure 6 - Main sequences of the East Carpathian Area (graphic symbols as in fig. 2).

Abbreviations. $H$ : Holocene; TGL : late glacial; $L$ : loess; $L S$ : sandy loess; $L m$ : loam; $S L$ : silty sand; $S$ : sand; hum : humiferous horizons; gl $t$ : tundra gley; MG : Malu Galben; Mol : Molodova V; Cos : Cosautsi; Arch : archaeology;

Paléoenviron : palaeoenvironment; $P$ : periglacial, with deep frost or permafrost conditions; $A$ : arctic; $S A$ : subarctic; $B$ : boreal. 
MOLODOVA V (Ukraine) MITOC (Roumanie) COSAUTSI (Moldavie)

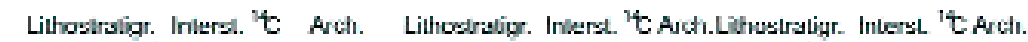

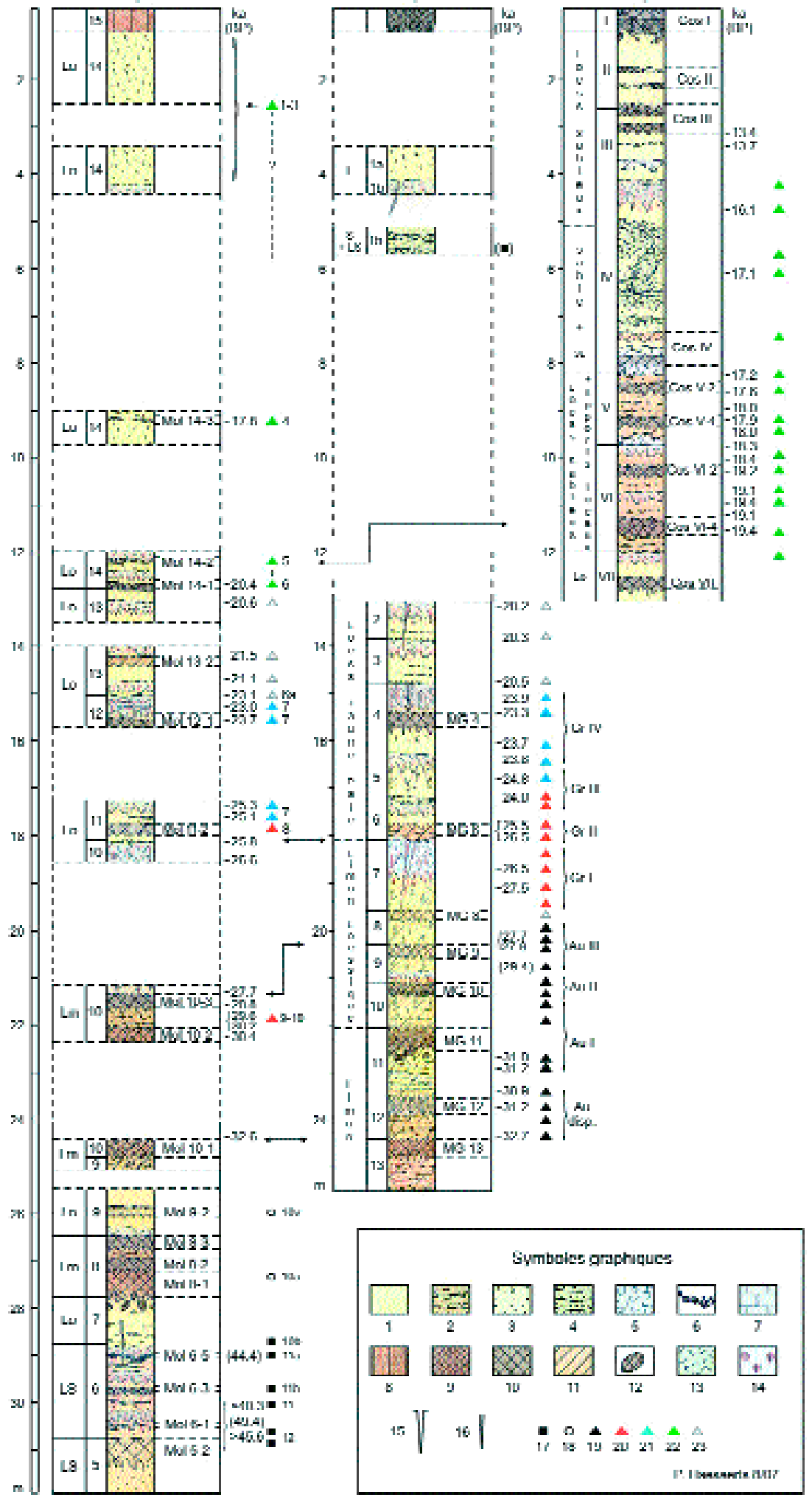

SEOUENCE REGIONALE

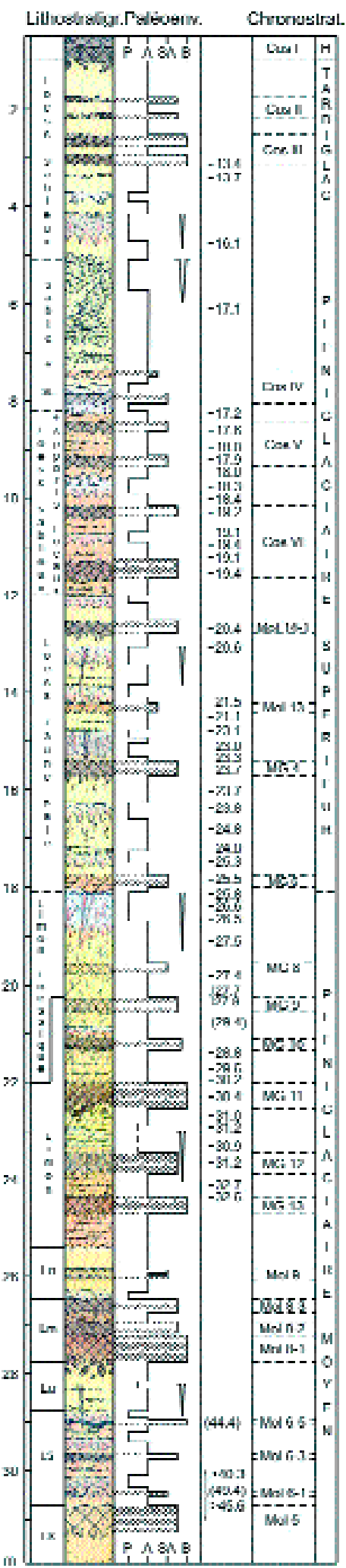

Figure 7 - La séquence est-carpatique de \pm 55000 à 10000 BP : schéma corrélatif (symboles graphiques : voir figure 2 ; abréviations : idem figure 6).

Figure 7 - The East Carpathian sequence from $\pm 55,000$ to 10,000 BP: correlation scheme (graphic symbols as in figure 2; abbreviations: see figure 6). 
mais surtout la succession des événements climatiques à différentes échelles de temps. Il apparaît donc que les événements climatiques spécifiques des différentes périodes sont exprimés de manière similaire dans les deux séquences régionales, même lorsqu'il s'agit d'oscillations de courte durée. C'est le cas, par exemple, de l'épisode interstadiaire Malu Galben 6, voisin de 25500 BP, qui est enregistré à la base de la couverture loessique du pléniglaciaire supérieur à Molodova $\mathrm{V}$ et à Mitoc, mais aussi à Willendorf et à Pavlov (fig. 8). De la même manière, les courts épisodes interstadiaires Molodova 14-1, Cosautsi VI-4 et Cosautsi VI-2 entre 20400 et 19000 BP, ont leurs équivalents à Grubgraben en Basse Autriche. Le système fonctionne également dans le cas des péjorations climatiques, notamment celles associées aux gleys de toundra G1 à G7 de Dolní Vestonice qui occupent des positions similaires dans les deux séquences régionales. De la sorte, il est possible de reconnaître des épisodes climatiques d'une durée de quelques siècles supposés synchrones à l'échelle du domaine loessique d'Europe centrale, avec un degré de résolution sensiblement supérieur à celui des dates radiométriques.

La séquence globale établie ci-dessus pour la période 33000 - 10000 BP fournit donc un canevas stratigraphique extrêmement précis autorisant une restitution objective de la distribution dans l'espace et dans le temps des différents ensembles culturels du Paléolithique supérieur dans un cadre paléoenvironnemental bien défini, avec un degré de résolution de l'ordre de quelques siècles dans la plupart des cas. Cette démarche concerne en priorité les gisements auxquels nous avons eu accès et intègre principalement des données stratigraphiques et chronologiques de première main (Damblon et al. 1996 ; Damblon \& Haesaerts 1997 ; Haesaerts et al. 1996, 2003) ; elle reprend également un certain nombre d'éléments discutés dans les différentes synthèses sur le Paléolithique supérieur (Kozlowski 1996, 1998 ; Djindjian et al. 1999 ; Svoboda 2000 ; Djindjian 2002 ; Otte \& Noiret 2004), synthèses dont le cadre chronostratigraphique repose le plus souvent sur des âges radiométriques déconnectés des données stratigraphiques.

\section{5 - LA SÉQUENCE ARCHÉOLOGIQUE}

\section{1 - L’Aurignacien}

En Basse Autriche, la séquence aurignacienne débute, semble-t-il, au cours de l'interstade de Schwallenbach I avec la couche culturelle 3 de Willendorf datée 38880 et 37930 BP (Haesaerts et al. 1996 ; Haesaerts \& Teyssandier 2003), laquelle est probablement contemporaine du Bohunicien en Moravie (Svoboda 2001). Quant à l'Aurignacien classique, il est surtout bien documenté entre \pm 32500 et $\pm 29000 \mathrm{BP}$, période qui couvre notamment les interstades Schwallenbach II et Schwallenbach III (fig. 4). A l'est des Carpates, c'est principalement la séquence de Mitoc-Malu Galben qui sert de référence avec une succession d'ateliers aurignaciens compris entre \pm 32700 et \pm 27 $500 \mathrm{BP}$, lesquels encadrent les épisodes interstadiaires Malu
Galben 12 à 9 (fig. 6 à 8). L'Aurignacien était également bien représenté à Ripiceni-Izvor sur la rive roumaine du Prut en aval de Mitoc (Paunescu 1993) mais la stratigraphie de ce site, actuellement noyé sous les eaux d'un lac de barrage, n'a pu être intégrée dans la séquence régionale.

\section{2 - Le Gravettien ancien et moyen}

Première période (de \pm 30500 à $28000 \mathrm{BP}$ ) - Un point important qui concerne directement la problématique de l'origine du Gravettien en Europe centrale, porte sur la position de la couche culturelle 5 de Willendorf dans un horizon humifère daté $30500 \mathrm{BP}$ et rapporté à l'interstade Schwallenbach III (Haesaerts 1990b ; Haesaerts et al. 1996). Cette attribution, mise en doute sur la base de données partielles par F. Djindjian et al. (1999 : 400), repose cependant sur l'intégration des observations récentes et des levés des fouilles anciennes dans un système stratigraphique et chronologique bien documenté que viennent renforcer la stratigraphie et les dates radiocarbonne du site adjacent de Schwallenbach (Haesaerts et al. 1996 ; Haesaerts \& Teyssandier 2003). Cet ensemble de données atteste donc la présence du Gravettien ancien à Willendorf dès $30500 \mathrm{BP}$ dans un contexte régional nettement aurignacien lequel va subsister jusque $\pm 28400 \mathrm{BP}$ à Albemdorf en Basse Autriche et jusque $\pm 27500 \mathrm{BP}$ dans le nord-est de la Roumanie (unité 8 à Mitoc) et peut-être même au-delà dans certains sites de Moldavie (Borziac 1994 ; Noiret 2004). L'âge voisin de 30500 BP pour la couche 5 de Willendorf est également cohérent avec le contexte stratigraphique des couches 10 et 9 de Molodova $\mathrm{V}$ à nette composante gravettienne (Otte 1981; Chemysh 1987 ; Noiret 2004). Celles-ci constituent un ensemble bien individualisé, daté entre 30200 et 28730 BP, dont la position au début de l'oscillation froide qui suit l'épisode interstadiaire Malu Galben 11 est bien assurée (fig. 6 et 8). Enfin, ce schéma est également en bon accord avec l'âge de 29200 BP obtenu pour les premières occupations gravettiennes à Geissenklösterle dans le Jura Souabe (Conard et Bolus 2003).

Quant aux couches culturelles plus récentes, attribuées à la phase initiale du Gravettien entre 29000 et 28000 BP par F. Djindjian et al. (1999), elles concernent principalement de petites concentrations atypiques; celles-ci sont datées 28 560 BP à Willendorf au-dessus de la couche 5 et 28220 BP dans la briqueterie à Dolní Vestonice (Haesaerts 1990a). Dans ce contexte, il faut aussi mentionner la couche culturelle solifluée de la station A à Dolní Vestonice I supposée représenter l'occupation la plus ancienne du site (Klíma 1963), dont la position par rapport au sol interstadiaire W $2 / 3$ et par rapport aux charbons de bois datés $29300 \mathrm{BP}$ demeure problématique (Oliva 2000). De même, la date de 28950 BP obtenue à Mitoc-Malu Galben pour l'atelier gravettien à la base de l'unité 7 demande à être confirmée car nettement trop vieille par rapport à la chronologie de la séquence locale (Damblon et al. 1996).

Deuxième période (de 28000 à 26000 BP) - Cette seconde période, qui enregistre la dégradation climatique de la phase terminale du pléniglaciaire moyen, correspond 
également au plein développement du Gravettien moyen et du Pavlovien (Svoboda et al. 1994, 2000 ; Oliva 2000 ; Otte \& Noiret 2004). A Dolní Vestonice II, les occupations associées aux concentrations A-B-C débutent vers 27500 BP (Klíma 1995). De même, les chasseurs du Gravettien moyen étaient présents à Krems-Hundssteig vers 27940 BP (Neugebauer-Maresch, 2002) mais aussi à Mitoc-Malu Galben sur le Prut vers 27000 BP (base de l'unité 7) et probablement à Mejigirzi sur le Dniestr moyen (Koulakovska \& Otte 1998) où la couche principale a été datée récemment 27070 BP sur bois carbonisé (Haesaerts et al. 2004 : 45). Quant aux grandes occupations du Pavlovien datées majoritairement entre 26500 et 25500 BP à Dolní Vestonice, à Pavlov et à Predmostí, elles encadrent la péjoration climatique associée au gley de toundra G1 qui termine le pléniglaciaire moyen (fig. 4 et 8). C'est également le cas de la couche 6 de Willendorf, de l'occupation principale d'Aggsbach et des premiers grands ateliers gravettiens de Mitoc-Malu Galben datés entre 26450 et 25500 $\mathrm{BP}$, lesquels occupent une position similaire dans la partie sommitale du gley de toundra et à son interface avec la couverture loessique du pléniglaciaire supérieur.

Dans ce contexte, la concentration des habitats du Pavlovien autour de sites occupant une position remarquable dans le paysage (Svoboda et al. 2000) paraît bien répondre à l'emprise des conditions rigoureuses spécifiques de l'extrême fin du pléniglaciaire moyen. C'est le cas notamment des occupations du Pavlovien à Dolní Vestonice où la présence d'accumulations, probablement naturelles, d'ossements de mammouth au pied des monts Pavlov serait lié à l'attrait que le contexte géologique de ce lieu aurait exercé sur les grands herbivores suite à des exigences spécifiques dues au stress lié aux conditions rigoureuses. En cela, les habitats pavloviens se distinguent des occupations gravettiennes et aurignaciennes antérieures, lesquelles paraissent relativement dispersées dans le paysage, associées à un contexte climatique plus diversifié et relativement humide avec couvert végétal en mosaïque de type steppe arborée (Svobodová \& Svoboda 1988 ; Rybnicková E. \& Rybnicek K. 1991 ; Svobodova 1991).

Troisième période (de 26000 à 25000 BP) - Pour terminer, il nous faut considérer ici un dernier ensemble d'occupations du Gravettien moyen contemporaines des premiers apports loessiques du Pléniglaciaire supérieur mis en place localement le long des vallées principales entre 26000 et 25000 BP. Cet ensemble, qui assure en quelque sorte la transition vers le Gravettien supérieur, regroupe les couches 7 et 8 de Willendorfet la couche 8 de Molodova V (fig. 7), les couches 8 de ces deux sites étant incorporées dans un sol humifère intra-loessique bien daté vers $25500 \mathrm{BP}$, également reconnu à Pavlov II. Le même ensemble comprend les occupations term inales du Pavlovien datées entre 25500 et 25000 BP à Dolní Vestonice, à Pavlov I et à Predmostí, ainsi que la couche principale de Milovice en Moravie et la partie inférieure de la concentration gravettienne II à Mitoc-Malu Galben.

\subsection{Le Gravettien supérieur}

Première période (de 25000 à 23000 BP) - En Europe centrale, la distribution de ce complexe techno-culturel caractérisé par des pointes à cran (Otte 1981 ; Kozlowski 1986), reposait classiquement sur six sites ou groupes de sites : soit, Spadzista (sous-unités $6 \mathrm{~b}$ et $6 \mathrm{a}$ ), Petrkovice, Moravany, Nitra Cermán, Wllendorf (couche 9) et Molodova V (couche 7). Depuis, s'y sont ajoutés MitocMalu Galben (Gravettien IV : Otte et al. 1996) et probablement Dolní Vestonice III (Skrdla et al. 1996). L'ensemble est classiquement situé entre 24500 et 22000 BP (Kozlowski 1986, 1996 ; Djindjian et al. 1999), mais les nouvelles données de Willendorf, Molodova et Mitoc permettent de préciser cette chronologie (Haesaerts et al. 1996, 2003).

En particulier, le développement du Gravettien à pointes à cran en Europe centrale paraît bien contemporain du dépôt de la première couverture loessique du pléniglaciaire supérieur dans un environnement froid mais encore légèrement humide, ce dont témoignent les gleys de toundra qui y sont associés. Les premiers témoins de cette industrie apparaissent vers $25000 \mathrm{BP}$ de manière quasi synchrone de part et d'autre des Carpates. A Willendorf, la couche culturelle 9 fut datée 24910 BP sur la partie centrale d'un os long provenant des fouilles de J. Bayer (1930); notons à ce propos que les âges compris entre 24370 et 23180 BP mentionnés par J. Kozlowski (1998: 131) et J. Svoboda 2000 : 200) pour la couche 9 sont non valides car ils se réfèrent à des dates obtenues sur la partie externe de l'os long et sur un fragment d'omoplate de mauvaise qualité de la même couche (Haesaerts et al. 1996 : 38). A Molodova V, la partie principale de la couche 7 fouillée par A. Chernysh (1959), située avec précision dans la sous-unité 11-3 dans les profils ouverts en 1997-98 (fig. 6), a fourni trois dates sur charbon de bois entre 25280 et 25130 BP (Haesaerts et al. 2003); par contre, la partie supérieure de la couche 7 située par I. Ivanova (1987) dans l'horizon humifère sus-jacent (sousunité 12-1) et dans le gley de toundra 12-2, fut datée respectivement $23650 \mathrm{BP}$ et $23000 \mathrm{BP}$ (fig. 6 et 7).

A Mitoc, mais aussi à Dolní Vestonice III, à Moravany-Lopata et à Spadzista, le Gravettien à pointes à cran est attesté entre 24780 et 23000 BP, les principales occupations de Spadzista datées vers 23000 BP se situant juste en dessous et dans le sommet soliflué du gley de toundra principal (sous-unités $6 \mathrm{a}$ et $6 \mathrm{~b}$ ). A cet ensemble appartiennent également l'occupation de Petrkovice, dont la date 23370 BP obtenue récemment nous parait plus fiable que celle de 20 790 BP issue des fouilles antérieures (Jarosová et al. 1996), ainsi que l'occupation de Nitra Cermán, datée 23000 BP sur charbon de bois, qui est présente dans la partie basale de la couverture loessique supérieure (Bárta 1980).

Deuxième période (de 23000 à $\pm 20000 \mathrm{BP}$ ) - La mise en place de la couverture loessique supérieure dans un contexte froid, mais surtout très sec sur la base de la malacologie (Haesaerts et al. 2003 : 175), a été datée à Molodova entre 23000 et $\pm 20000 \mathrm{BP}$, période correspondant à l'âge du stade glaciaire de BrandenbourgLezsno dans la nord de l'Europe (Kozarski 1980). A l'ouest 


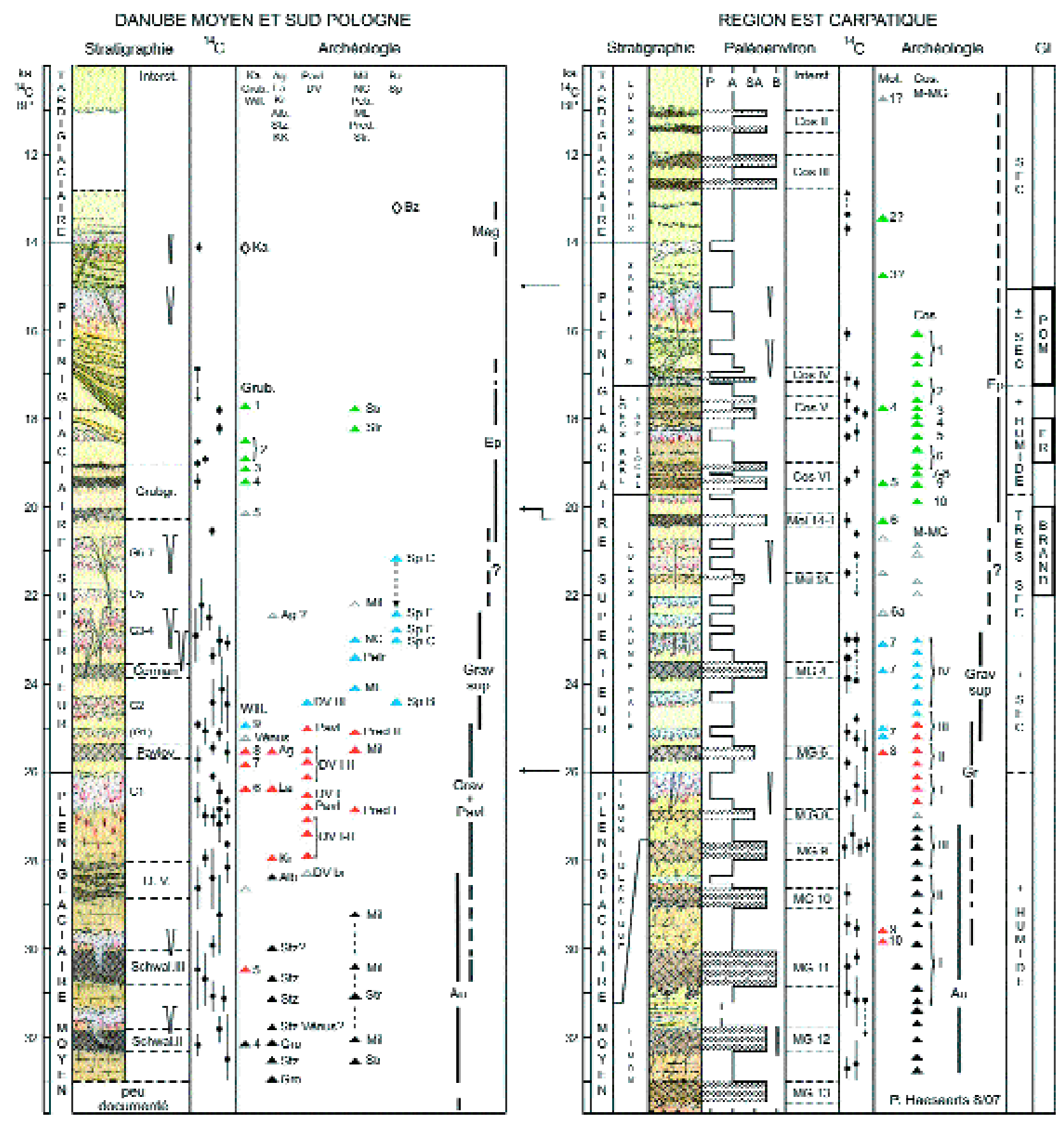

Figure 8 - Schéma corrélatif des séquence loessiques du Danube moyen et du domaine est-carpatique (symboles graphiques : voir figure 2 ; abréviations : idem figures 4 et 6).

Figure 8 - Correlation scheme of the loess sequences from Middle Danube and East Carpathian Area (graphic symbols as for figure 2; abbreviations: see figures 4 and 6). 
des Carpates, la chronologie des premiers apports éoliens de cette importante phase loessique demeure imprécise en raison de la fiabilité aléatoire des âges sur os en milieu loessique, du moins à l'échelle du millénaire (Damblon et al. 1996), et du manque de charbon de bois dans les dépôts de cette période. Ces loess n'ont livré que de rares assemblages atypiques témoignant d'occupations occasionnelles et de courte durée. C'est le cas du niveau supérieur d'Aggsbach et de Milovice vers 22500 BP, ou encore d'ateliers de taille isolés comme ceux de la partie basale de la couche 5 à Spadzista $C$ attribués à une phase tardive du Gravettien supérieur (Kozlowski \& Sobczyk 1987 ; Kozlowski 1998 ; Escutenaire et al. 1999).

A l'est des Carpates, le long du Prut et du Dniestr, les occupations gravettiennes bien documentées font également défaut dans les loess de cette période ; ceux-ci incorporent cependant un grand nombre de petites concentrations lithiques ou de pièces dispersées associées à des restes de renne et de cheval, avec localement présence de charbon de bois. A Molodova $\mathrm{V}$ par exemple, ces petites concentrations réparties sur plusieurs niveaux dans les loess de l'unité 13 , furent datés respectivement 23120 BP à la base, 21500 BP dans la partie médiane et $20600 \mathrm{BP}$ vers le haut de l'unité (fig. 6). En d'autres termes, compte tenu de l'exceptionnel potentiel archéologique des vallées du Prut et du Dniestr dont seule une infime partie fut exploitée, ces quelques témoins attestent la présence répétée de petits groupes de chasseurs dans la région au cours de cette période froide mais surtout très sèche comprise entre 23000 et $\pm 20000 \mathrm{BP}$. Localement, des indices d'occupations plus importantes existent néanmoins dans les dépôts de cette période, par exemple dans la partie inférieure du loess poudreux de Crasnaleuca au nord de Mitoc mais celles-ci n'ont pas encore fait l'objet d'études détaillées (Chirica 1989).

En conséquence, la période correspondant à la seconde génération loessique, que l'on a souvent associée à un vide d'occupation en relation avec le maximum glaciaire du stade Brandenburg-Lezsno (Kozlowski 1996, 1998 ; Djindjian et al. 1999 ; Djindjian 2002), paraît surtout enregistrer un changement majeur du mode de subsistance des populations de chasseurs collecteurs après $23000 \mathrm{BP}$; celui-ci s'accompagne, semble-t-il, d'une réorientation des pratiques cynégétiques vers la chasse saisonnière du renne et du cheval en réponse à une modification importante de l'environnement liée à l'extension considérable des substrats xériques et à l'uniformisation des biotopes steppiques peu favorables à la grande faune autochtone (Haesaerts 1990b).

\section{4 - L’Epigravettien et les faciès associés}

Première période (de \pm 20000 à $\pm 17000 \mathrm{BP}$ ) - Les industries de cette période se réfèrent à deux groupes d'occupations : celui de Grubgraben (couches culturelles 5 à 1) en Basse Autriche et celui de Cosautsi (couches 10 à 1d) et de Molodova (couches 6 à 4) sur le Dniestr (fig. 2,6 et 8 ). Dans les deux régions, ces occupations accompagnent une succession de courtes oscillations intersta- diaires et d'épisodes plus froids dans un contexte climatique relativement humide. Cette succession constitue en quelque sorte la signature de la seconde moitié du pléniglaciaire supérieur entre \pm 20000 et $\pm 17000 \mathrm{BP}$, une période également associée à l'extension maximum de l'inlandsis scandinave dans le nord de l'Europe (fig. 8).

A Grubgraben, les couches culturelles 4 et 3 datées 19380 et $18920 \mathrm{BP}$ et les deux horizons humifères associés, ainsi que l'horizon humifère inférieur qui contient les éléments de la couche 5, se rapportent à un épisode complexe dénommé «phase climatique de Grubgraben». Les couches culturelles $2 a$ et $2 b$ datées 18890 et $18380 \mathrm{BP}$, accompagnent les loess sus-jacents, tandis que la couche 1 est associée à une phase d'arrêt des apports loessiques antérieure à 16 800 BP (Haesaerts 1990c). L'industrie lithique des couches culturelles 5 à 1 constitue un ensemble cohérent rapporté à l'Epigravettien par A. Montet-White (1990), mais qui présenterait cependant certains caractères «aurignacoïdes» (Brandtner 1996 ; Street \& Terberger 1999). A cet ensemble, appartiendraient également les occupations de Langsmannersdorf et Rosenburg en Basse Autriche, deux gisements dépourvus de contexte stratigraphique, datés sur os entre 20580 et 20120 BP (Neugebauer-Maresch 1999). De même, les occupations épigravettiennes rapportées à la période 18900 - $17700 \mathrm{BP}$ à Sagvar en Hongrie (Gabori 1965) et à Stránská Skála en Moravie (Svoboda et al. 1994) sont probablement contemporaines des couches 3,2 et 1 de Grubgraben.

A l'est des Carpates, ce sont les séquences de Molodova $\checkmark$ et surtout de Cosautsi qui sont les mieux documentées ; elles intègrent un grand nombre de couches épigravettiennes riches en charbon de bois, datées entre 20400 et $17200 \mathrm{BP}$, qui paraissent bien s'inscrire en continuité avec les occupations occasionnelles de la période antérieure (Noiret 2004). A Molodova V, où les Epigravettiens sont présents dès $20400 \mathrm{BP}$, la couche 6 couvre une large superficie et se trouve associée à un horizon humifère interstadiaire bien exprimé (sous-unité 14-1) qui établit la liaison avec la longue série d'occupations correspondant aux couches culturelles 10 à $1 \mathrm{~d}$ de Cosautsi. Celles-ci se distribuent en semi-continuité autour de trois groupes d'horizons humifères rapportés aux épisodes interstadiaires Cosautsi VI-4 et VI-2 (entre 19400 et 19000 BP), Cosautsi V-4 et V-2 (entre 18000 et 17500 BP) et Cosautsi IV-4 et IV-3 (vers 17200 BP), lesquels sont séparés par deux péjorations climatiques situées vers 18200 et 17200 BP probablement contemporaines des stades glaciaires de Frankfurt-Posnan et de Poméranie (fig. 8). D'autres témoins d'occupations épigravettiennes de cette période sont préservés à Molodova $\mathrm{V}$ (couches 5 et 4), à Korman IV (Ivanova 1977 ; Noiret 2004), à Podgor au voisinage de Cosautsi (Borziac 1994), mais aussi à Crasnaleuca le long du Prut (Chirica 1989).

Deuxième période (de \pm 17000 à $\pm 14500 \mathrm{BP})-\mathrm{En}$ Europe centrale, cette période qui correspond au stade glaciaire de Poméranie, se caractérise par des conditions climatiques extrêmes avec plusieurs épisodes de permafrost et une prédominance des faciès sableux souvent 
soufflés à partir des plaines alluviales avoisinantes ; elle s'accompagne d'une nouvelle césure dans la séquence du Paléolithique supérieur, les dépôts correspondant étant généralement stériles. Toutefois, le bassin du Dniestr fait à nouveau exception, principalement à Cosautsi (couches 1c à 1a) et peut-être aussi à Korman IV, avec de petites concentrations lithiques peu différenciées mais encore attribuées à l'Epigravettien, datées entre 17100 et 16050 BP (Ivanova 1977 ; Noiret 2004). Celles-ci y sont préservées dans les dépôts sableux et sablo-limoneux antérieurs à l'épisode rigoureux avec gley de toundra et fentes de gel qui termine le pléniglaciaire supérieur (fig. 7 et 8).

Troisième période (de \pm 14500 à $\pm 10000 \mathrm{BP}$ ) - Le Tardiglaciaire est associé à la phase finale du Paléolithique supérieur dans un environnement climatique contrasté avec plusieurs phases plus sèches dont témoigne une double génération d'apports éoliens qui encadrent les sols humifères du complexe Bölling-Alleröd préservés dans les parties basses du paysage. Dans le domaine occidental, les rares gisements connus appartiennent au complexe magdalénien. C'est le cas de la couche culturelle magdalénienne de Kamegg dans la vallée de la Kamp, datée $14100 \mathrm{BP}$ sur os à la base d'un loess comparable à celui de la séquence supérieure de Grubgraben (Haesaerts et al. 2004). De même, l'industrie magdalénienne de Brzoskwinia, dans le sud de la Pologne, préservée dans les sables de couverture du Dryas le plus ancien, peut être mise en parallèle avec le Magdalénien supérieur de la grotte de Pekarna en Moravie (Svoboda et al. 1994).

Par contre, à l'est des Carpates, la situation est plus complexe. Alors qu'en Ukraine centrale, la présence de l'Epigravettien est attestée entre 15000 et 13500 BP, par les célèbres campements à structure d'habitat en ossements de mammouth (Soffer 1985 ; lakovleva 2001), au contraire, dans les bassins du Dniestr et du Prut, l'attribution chronologique des petites concentrations épigravettiennes préservées dans la partie supérieure de la couverture loessique demeure problématique, en particulier pour les couches 3 à 1 de Molodova V.

\section{6 - VUE D'ENSEMBLE}

La séquence interrégionale élaborée à l'échelle du domaine loessique d'Europe centrale pour la période 33000 - 10 $000 \mathrm{BP}$, associe les données pédostratigraphiques, les enregistrements paléoclimatiques et chronologiques et les données archéologiques. Ce système bien documenté permet ainsi de préciser les liens entre ces différentes composantes. Dans ce contexte, la chronologie des événements constituait un objectif prioritaire ; elle repose sur une séquence complexe et reproductible de courts épisodes climatiques dont la chronologie fut fixée par de longues séries de datations ${ }^{14} \mathrm{C}$ cohérentes obtenues pour la plupart sur charbon de bois, ce qui autorise le positionnement de la majorité des événements dans l'échelle du temps avec un degré de résolution de l'ordre de quelques siècles.
Par ailleurs, l'insertion dans ce système d'un grand nombre de sites et d'horizons d'occupations du Paléolithique supérieur bien positionnés en stratigraphie, a permis de démontrer le caractère synchrone de la distribution des principaux ensembles techno-culturels de part et d'autre des Carpates, compte tenu de la marge d'imprécision inhérente au schéma chronologique (fig. 8). Dès lors, cette approche s'inscrit en complément des différents essais de synthèse publiés ces demières années pour le Paléolithique supérieur d'Europe centrale (Kozlowski 1996, 1998 ; Djindjian et al. 1999 ; Svoboda 2000 ; Djindjian 2002 ; Otte \& Noiret 2004)

Un autre aspect spécifique de la séquence interrégionale concerne l'incidence des variations du climat et de l'environnement sur le schéma évolutif du Paléolithique supérieur dans le domaine loessique d'Europe centrale au cours de la période considérée, un thème qui n'a guère été développé dans les essais de synthèse précédents. De fait, le degré de résolution du système a permis de mettre en évidence un remarquable parallélisme entre l'évolution de l'environnement et celle des ensembles techno-culturels ; il apparaitt en outre que ces deux processus évolutifs traduisent une succession rythmique des événements selon une périodicité de l'ordre de \pm 2500 ans.

Ce parallélisme se marque dès la fin du pléniglaciaire moyen avec la phase d'extension du Pavlovien et du Gravettien moyen entre 27500 et 25500 BP, dans un contexte climatique essentiellement rigoureux et humide qui contraste avec la succession récurrente d'épisodes interstadiaires de la période antérieure. II se poursuit au pléniglaciaire supérieur avec la mise en place d'une première couverture loessique entre \pm 25500 et $23000 \mathrm{BP}$ dans un environnement encore relativement humide; c'est au cours de cette période que se développe le Gravettien supérieur à pointes à cran, et cela en continuité avec les occupations gravettiennes antérieures (Svobodá et al. 2000). De même, la diminution considérable des occupations entre \pm 23000 et $\pm 20000 \mathrm{BP}$, qui caractérise la mise en place de la seconde génération loessique associée au stade glaciaire Brandenbourg-Lezslo, répond probablement à une uniformisation des biotopes steppiques peu favorables à la grande faune d'herbivores autochtones suite à l'extension considérable des substrats xériques.

Cette situation se maintient au cours de la seconde partie du pléniglaciaire supérieur, avec toutefois une plus grande densité de campements saisonniers récurrents au cours de la phase plus humide comprise entre \pm 20000 à \pm 17000 BP. En Basse Autriche, Grubgraben et les sites adjacents évoquent un environnement steppique (cf. § 2.2), tandis que dans le domaine est-carpatique, les données palynologiques et l'abondance de charbon de bois dans les gisements épigravettiens à Cosautsi, Molodova et Korman IV, laissent supposer la présence de parcelles boisées le long des vallées principales dans un paysage de forêt-steppe (cf. § 3.3). Néanmoins, au cours de cette période, la large distribution des substrats loessiques demeure un facteur déterminant de part et d'autre des Carpates et se répercute sur le mode de subsistance des populations, surtout 
axé sur les migrations saisonnières du renne et du cheval, ce dont témoigne la composition des faunes de chasse de la plupart des gisements. En cela, elles se distinguent des faunes antérieures à $23000 \mathrm{BP}$, lesquelles sont nettement plus diversifiées et le plus souvent composées de grands herbivores autochtones. De fait, entre \pm 20000 et \pm 17000 $\mathrm{BP}$, la plupart des gisements se situent le long des principales voies de migration du renne. Grubgraben fait face à la vallée du Danube, à hauteur du débouché de la rivière Kamp qui donne accès, vers le nord, au plateau morave et à la plaine baltique. De même, la vallée du Dniestr et ses abords constituaient un parcours de migration obligé entre les zones marécageuses du Pripet au nord et les steppes méridionales à proximité de la mer Noire actuelle.

Enfin, la demière phase rigoureuse du pléniglaciaire supérieur entre 17000 et $\pm 14500 \mathrm{BP}$ voit à nouveau diminuer considérablement les occupations de part et d'autre des Carpates. Cette situation se modifie à nouveau au début du Tardiglaciaire, une période caractérisée par une différenciation régionale marquée des complexes techno-culturels du domaine loessique en Europe centrale (Otte \& Noiret 2004).

En conclusion, les différents stades évolutifs du Paléolithique au cours du pléniglaciaire supérieur semblent surtout induits par les modifications de l'environnement climatique à l'échelle de l'Europe centrale et par l'impact de l'extension des couvert u res loessiques sur la composition de la grande faune liée à la nature et à la diversité du paysage végétal. Par contre, au cours d'une même phase climatique, la fréquence et le type d'occupation s'avèrent indépendantes des oscillations climatiques de courtes durées (fig. 7 et 8). Dans ce contexte, les nouvelles données réunies en Europe centrale pour le pléniglaciaire supérieur conduisent à nuancer quelque peu l'hypothèse d'un vide d'occupation entre 21000 et 18000 BP avancée précédemment par divers auteurs (Soffer 1985 ; Kozlowski 1996, 1998 ; Djindjian 2002). De fait, les gisements du domaine occidental et du domaine est-carpatique illustrent la variabilité dans le temps des environnements climatiques, mais aussi les diversités régionales du domaine loessique au cours de cette période généralement associée au maximum glaciaire. En particulier, les séquences de Mitoc, de Molodova, de Ko rman IV et de Cosautsi attestent la présence répétée des chasseurs paléolithiques dans la région pendant la quasi-totalité du pléniglaciaire supérieur, les vallées du Prut et du Dniestr constituant une voie de migration obligée pour les hardes de rennes.

Nous sommes cependant conscients du fait que les schémas de distribution régionaux soulignant le synchronisme des différents ensembles techno-culturels du Paléolithique supérieur de part et d'autre des Carpates demeurent incomplets. Cela tient en particulier au nombre limité de sites pris en compte par rapport au potentiel supposé des régions concernées et à la part de hasard conditionnant leur découverte, une situation qui rend aléatoire les modèles de migration des populations du Paléolithique supérieur dans la Grande Plaine européenne (Mussi et al. 2000).

\section{Remerciements}

Ce travail représente l'aboutissement d'une démarche entamée au début des années quatre-vingt à l'initiative de Jean de Heinzelin avec comme point de référence les travaux de Bohuslav Klíma consacrés à Dolní Vestonice. Nous sommes également redevables aux collègues de Belgique, d'Allemagne et d'Europe centrale pour les conseils, les échanges d'informations et les discussions f ructueuses qui ont contribué à la bonne fin de cet article. Le financement de ces recherches a été assuré par les projets Sc-004 et Sc-09 de l'Etat belge, Service des Affaires Scientifiques, Techniques et Culturelles (SSTC) et par les projet INTAS 93-1693, INTAS 96-0072 et INTAS 2000-879.

\section{BIBLIOGRAPHIE}

BACHNER M., MATEICIUCOVA I. \& TRNKA G. 1996 - Die Spätaurignacien-Station Albemdorf im Pulkautal, NÖ. In: Svoboda J. (Ed), Palaeolithic in the Middle Danube Region; Anniversary volume to Bohuslav Klíma. Institute of Archaeology, ASCR (Brno): 93-119.

BÁRTA J. 1980 - Importants sites paléolithiques de la Slovaquie centrale et occidentale. Institut d'Archéologie de l'Académie Slovaque des Sciences (Nitra).

BAYER J. 1930 - Die Venus II von Willendorf. Eiszeit und Urgeschichte (Leipzig) 7: 48-60.

BORZIAC I. 1991 - Quelques données préalables sur l'habitat tardi-paléolithique pluristratifié de Cosseoutsy sur le Dniestr Moyen. In: Chirica V. \& Monah D. (Eds), Le Paléolithique et le Néolithique de la Roumanie en contexte européen. Bibliotheca Archaeologica lassiensis (Jassy) 4: 56-71.

BORZIAC I. 1993 - Les chasseurs de renne de Kosoioutsy, site paléolithique tardif à plusieurs niveaux sur le Dniestr moyen (rapport préliminaire). L'Anthropologie (Paris) 97 (23): 331-336.

BORZIAC I. 1994 - Paleolithic and the Mesolithic in the Dnjestr - Pruth Area of Moldova (en roumain). Traco-Dacica (Bucarest) 15 (1-2): 19-40.

BRANDTNER F. 1996 - Zur geostratigraphischen und kulturellen Zuordnung der Paläolithstation Grubgraben bei Kammern, NÖ. In: Svoboda J. (Ed), Palaeolithic in the Middle Danube Region; Anniversary volume to Bohuslav Klíma. Institute of Archaeology, ASCR (Brno): 121-145.

CHERNYSH A.P. 1959 - The Upper Paleolithic of the Middle Dniester Area (en russe). In: Gromov V.L. \& Okladnikov A.P. (Eds.), Palaeolithic of the Middle Dniester Area. Trudy Komissii po izucheniyu chetvertichnogo perioda (Moscou) 15: 5-214.

CHERNYSH A.P. 1987 - The standard multilayerd site Molodova V. Archeology (en russe). In: Ivanova I.K. \& 
Tzeitlin S.M. (Eds.), The multilayerd Palaeolithic Site Molodova $V$. The stone Men and environment. Nauka (Moscou): 7-93.

CHIRICA V. 1989 - The Gravettian in the East of the Romanian Carpathians. Bibliotheca Archaeologica lassiensis (Jassy) 3

CHIRICA V. 2001 - Gisements paléolithiques de Mitoc. Le Paléolithique Supérieur de Roumanie à la lumière des découvertes de Mitoc. Editions Hélios (Jassy).

CONARD N. \& BOLUS M. 2003 - Radiocarbon dating the appareance of modern humans and timing of cultural innovations in Europe: new results and new challenges. Journal of Human Evolution 44: 331-371.

DAMBLON F. \& HAESAERTS P. 1997 - Radiocarbon chronology of representative Upper Palaeolithic sites in the Central European Plain: a contribution to the Sc-004 project. Préhistoire Européenne (Liège) 11: 255-276.

DAMBLON F. \& HAESAERTS P. 2002 - Anthracology and radiochronology of the Upper Pleistocene in the loessic areas of Eurasia. In: Thiébault S. (Ed), Charcoal Analysis. Methodological Approaches, Palaeoecological Results and Wood Uses. Proceedings of the 2nd International Meeting Anthracology, Paris, September 2000. BAR Intemational Series (Oxford) 1063: 65-71.

DAMBLON F., HAESAERTS P. \& VAN DER PLICHT J. 1996 New datings and considerations on the chronology of Upper Palaeolithic sites in the Great Eurasiatic Plain. PréhistoireEuropéenne (Liège) 9: 177-231.

DEMEK J. \& KUKLA J. 1969 - Periglazialzone, Löss und Paläolithikum der Tschechoslowakei. Tschechoslowakische Akademie der Wissenschaften, Geographisches Institut in Brno (Bmo).

DJINDJIAN F. 2002 - Ruptures et continuités dans les industries du maximum glaciaire en Europe centrale et orientale: la question de l'Epigravettien. In: Sinitsyn A., Sergin V. \& Hoffecker J. (Eds), Trends in the Evolution of the East European Palaeolithic. Kostienki in the context of the Palaeolithic of Eurasia, Ser. Research (Saint-Pétersbourg) 1: 250-255.

DJINDJIAN F., KOZLOWSKI J.K. et OTTE M. 1999 - Le Paléolithique supérieur en Europe. A. Colin, Paris.

ESCUTENAIRE C., KOZLOWSKI J.K., SITLIVY V. \& SOBCZYK K. 1999 - Les chasseurs de mammouths de la vallée de la Vistule. Krakóv-Spadzista $B$, un site gravettien à amas d'ossements de mammouths. Musées Royaux d'Art et d'Histoire et Université Jagellon de Cracovie. Monographie de Préhistoire Générale (Bruxelles) 4.

FINK J. 1969 - Le loess en Autriche. In: Stratigraphie des loess d'Europe. Supplément du Bulletin de l'Association Française pour l'Etude du Quaternaire (Paris) 17-21.
GABORI M. 1965 - Der zweite paläolithische Hausgrundriss von Sagvar. Acta Archaeologica Hungarica (Budapest) 17: 111-127.

HAESAERTS P. 1985 - Les loess du Pléistocène supérieur en Belgique. Comparaisons avec les séquences d'Europe centrale. Bulletin de l'Association Française pour l'Etude du Quatemaire (Paris) 22-23 (2-3): 105-115.

HAESAERTS P. 1990a - Evolution de l'environnement et du climat au cours de l'Interpléniglaciaire en Basse Autriche et en Moravie. In: Kozlowski J.K. (Ed), Les industries à pointes folliacées du Paléolithique supérieur européen, Cracovie. ERAUL (Liège) 42: 523-538.

HAESAERTS P. 1990b - Nouvelles Recherches au gisement de Willendorf (Basse Autriche). Bulletin de l'Institut royal des Sciences naturelles de Belgique, Sciences de la Tere (Bnxelles) 60: 203-218.

HAESAERTS P. 1990c - Stratigraphy of the Grubgraben loess sequence. In: Montet-White A. (Ed), Grubgraben, an open-air Epigravettian site in Lower Austria. Report for the 1987-1987 field seasons. ERAUL (Liège) 41: 14-35.

HAESAERTS P. \& TEYSSANDIER N. 2003 - The Early Upper Palaeolithic occupations of Willendorf II (Lower Austria): a contribution to the chronostratigraphic and cultural context of the beginning of the Upper Palaeolithic in Central Europe. In: Zilhao J. \& d'Errico F. (Eds), The Chronology of the Aurignacian and of the Transitional Technocomplexes. Dating. Stratigraphies, Cultural Implications. Proceedings of Symposium 6.1 of the XIVth Congress of the UISPP (University of Liège, Belgium, September 2-8,2001). Trabalhos de A rqueologia 33: 133-151.

HAESAERTS P. et VAN VLIET B. 1974 - Compte rendu de l'excursion du 25 mai 1974 consacrée à la stratigraphie des limons aux environs de Mons. Annales de la Société Géologique de Belgique (Liège) 97: 547-560.

HAESAERTS P., BORZIAC I., VAN DER PLICHT J. \& DAMBLON F. 1998 - Climatic events and Upper Palaeolithic chronology in the Dniestr Basin: new radiocarbon results from Cosautsi. In: Mook W. \& van der Plicht J. (Eds), Proceedings of the 16th International 14C Conference. Radiocarbon (Tucson, Arizona) 20 (2): 649-657.

HAESAERTS P., DAMBLON F., BACHNER M. \& TRNKA G. 1996 - Revised stratigraphy and chronology of the WIlendorf II sequence, Lower Austria. Archaeologia Austriaca (Vienne): 80: 25-42.

HAESAERTS P., BORZIAC I., CHIRICA V., DAMBLON F. \& KOULAKOVSKA L. 2004 - Cadre stratigraphique et chronologique du Gravettien en Europe centrale. In: Svoboda J. \& Sedlácková L. (Eds), The Gravettian along the Danube, $P$ roceedings of the Mikulov Conference, 20.-21. November 2002. The Dolní Vestonice Studies, Institute of Archaeology, ASCR (Brno) 11: 33-56. 
HAESAERTS P., BORZIAC I., CHIRICA V., DAMBLON F., KOULAKOVSKA L. \& Van DER PLICHT J. 2003 - The East carpathian loess record: a reference for the middle and late pleniglacial stratigraphy in Central Europe. Quatemaire (Paris) 14 : 163-188.

IAKOVLEVA L. 2001 - Recherches sur le Paléolithique supérieur de I'Ukraine (1997-2000). In: Noiret P. (Ed), Le Paléolithique supérieur européen. Bilan quinquennal 19962001. ERAUL (Liège) 97: 35-43.

IVANOVA I.K. 1977 - Geology and paleogeography of the site Korman IV on the general background of the geological history of the Paleolithic Middle Dniester Region (en russe). In: Goretski G.I. \& Tzeitlin S.M. (Eds.), The multilayer Paleolithic site Korman IV on the Middle Dniestr. Nauka (Moscou) : 126-181.

IVANOVA I.K. \& TZEITLIN S.M. 1987 - The multilayerd Palaeolithic Site Molodova V. The stone Age Man and environment (en russe). Nauka (Moscou).

JAROSOVÁ L., CILEK V., OCHES E. \& SNIESZKO Z. 1996 - Petrkovice, excavations 1994-1995. In: Svoboda J. (Ed), Palaeolithic in the Middle Danube Region; Anniversary volume to Bohuslav Klíma. Institute of Archaeology, ASCR (Bmo) : 191-206.

KLíMA B. 1963 - Dolní Vestonice. Vyzukum táboriste lovcu mamutu $v$ letech 1947-52. Monumenta archaeologica 11, Cekeslovenská Akademie Ved (Prague).

KLÍMA B. 1969 - Die grosse Anhäufung von Mammutknochen in Dolní Vestonice. Acta scientiarum naturalium, Academiae scientarum bohemoslovacae (Brno) 3 (6).

KLÍMA B. 1976 - Die paläolithische Station Pavlov II. Acta scientiarum naturalium, Academiae scientarum bohemoslovacae (Brno) 10.

KLÍMA B. 1995 - Dolní Vestonice II. Ein Mammutjägerrastplatz und seine Bestattungen. ERAUL (Liège) 73.

KOULAKOVSKA L. et OTTE M. 1998 - Mejigirzi. Préhistoire Européenne (Liège) 13: 149-166.

KOZARSKI S. 1980 - An outline of the Vistulian stratigraphy and chronology of the Great Poland Lowland. In: Rozycki S.Z. (Ed), Quaternary Studies in Poland, Vistulian Stratigraphy Poland, 79, Polish Academy of Sciences (Varsovie-Poznan) 2 (1): 21-35.

KOZLOWSKI J.K. 1986 - The Gravettian in Central and Eastern Europe. In : Wendorf F. \& Close A., Advances in World Archaeology. Academic Press (Orlando) 5: 131-200.

KOZLOWSKI J.K. 1996 - The Danubian Gravettian as seen from the northern perspective. In: Svoboda J. (Ed), Palaeolithic in the Middle Danube Region; Anniversary volume to Bohuslav Klíma. Institute of Archaeology, ASCR (Bmo) : 11-22.
KOZLOWSKI J.K. 1998 - Taxonomic position of the site in the frame of the Central European Late Gravettian. In: Kozlowski J.K. (Ed), Complex of Upper Palaeolithic sites near Moravany, Western Slovakia. VOL. II MoravanyLopata II (Excavations 1993-1996) (Cracovie) : 131-135.

KOZLOWSKI J.K. \& SOBCZYK K. 1987 - The Upper Palaeolithic site Krakóv-Spadzista street C2. Zeszyty Naukowe UJ. Prace Archeologiczne 42 : 7-68.

MEDIANIK S.I. 1994 - Vegetation of Moldovia in the Late Paleolithic. Intemational Palynological Congress 1992, Aspects of Archaeological Palynology. Arizona State University 29 : 141-145.

MONTET-WHITE A. 1990 - Grubgraben, an open-air Epigravettian site in Lower Austria. Report for the 19871987 field seasons. ERAUL (Liège) 41.

MOROSAN N. 1938 - Le Pléistocène et le Paléolithique de la Roumanie du Nord-Est. (Les dépôts géologiques, leur faune, flore et produits d'industrie). Annuarul Institutului Geologie al României (Bucarest) 19.

MUSSI M., ROEBROEKS W. \& SVOBODA J. 2000 Hunters of the Golden Age: an introduction. In: Roebroeks W., Mussi M., Svoboda J. \& Fennema K. (Eds) Hunters of the Golden Age. The Mid Upper Palaeolithic of Eurasia 30,000 - 20,000 BP. University of Leiden (Leiden): 1-11.

NEUGEBAUER-MARESCH Ch. 1996 - Zu Stratigraphie und Datierung der Aurignac-Station am Galgenberg von Stratzing/Krems-Rehberg, NÖ. In: Svoboda J. (Ed), Palaeolithic in the Middle Danube Region; Anniversaryvolume to Bohuslav Klíma. Institute of Archaeology, ASCR (Brno) : 67-80.

NEUGEBAUER-MARESCH Ch. 1999 - Le Paléolithique en Autriche. Préhistoire d'Europe (Grenoble) 8.

NEUGEBAUER-MARESCH Ch. 2002 - Wege zur Eiszeit. Ein neues Projekt zur Altsteinzeitforschung der Prähistorischen Kommission der Östereichischen Akademie des Wissenschaften und des Fonds zur Förderung der wissenschaftlichen Forschung. Österreichischen Akademie der Wissenschaften, Anzeiger der philosophisch-historischen Klasse (Vienne)135 : 31-46.

NOIRET P. 2004 - Le Paléolithique supérieur de la Moldavie. L'Anthropologie (Paris) 108 : 425-470.

OLIVA M. 1989 -. La cabane des chasseurs de mammouth de Milovice (Moravie du Sud). L'Anthropologie (Paris) 93 (4) : 887-892.

OLIVA M. 2000 - Dolní Vestonice I. Une révision de la stratigraphie culturelle. Anthropologie (B mo) 38 (3) : 283-290.

OLIVA M. 2001 - Paléolithique supérieur dans les pays Thèques: bilan des travaux 1995-2000. In: Noiret P. (Ed), Le Paléolithique supérieur européen. Bilan quinquennal 19962001. ERAUL (Liège) 97: 59-72. 
OTTE M. 1981 - Le Gravettien en Europe Centrale. Dissertationes Archaeologicae Gandenses (Brugge) 20.

OTTE M. \& NOIRET P. 2004 - Evolution du Gravettien au Moyen Danube. In: Svoboda J. \& Sedlácková L. (Eds), The Gravettian along the Danube, Proceedings of the Mikulov Conference, 20.-21. November 2002. The Dolní Vestonice Studies, Institute of Archaeology, ASCR (Brno) 11 : 8-32.

OTTE M., LOPEZ-BAYON I., NOIRET P., BORZIAC I. et CHIRICA V. 1996 - Recherches sur le Paléolithique supérieur de la Moldavie. Anthropologie et Préhistoire (Bnxelles) $107:$ 1-36.

PAUNESCU A. 1993 - Ripiceni-Izvor. Paleolitic si Mezolitic, studiu monografi. Academia Romana; institutul de Arheologie «Vasile Parvan»; Biblioteca de arheologie (Bucarest) 52.

PAZDUR A. 1998 - Radiocarbone dating on bone samples. In: Kozlowski J.K. (Ed), Complex of Upper Palaeolithic sites near Moravany, Western Slovakia. VOL. II MoravanyLopata II (Excavations 1993-1996) (Cracovie) 127.

RYBNICKOVÁ E. \& RYBNICEK K. 1991 - The environment of the Pavlovian - palaeoecological results from Bulhary, South Moravia. In: Kovar-Eder (Ed), Palaeovegetational Development in Europe and Regions relevant to its Palaeofloristic Evolution (Vienne): 73-79.

SKRDLA P., CILEK V. \& PRICHYSTAL A. 1996 - Dolní Vestonice III, excavation 1993 - 1995. In: Svoboda J. (Ed), Palaeolithic in the Middle Danube Region; Anniversary volume to Bohuslav Klíma. Institute of Archaeology, ASCR (B mo) : 173-190.

SOBCZYK K. 1995 - Osadnictwo wschodniograweckie w dolinie Wisly pod Krakóvem Rozprawy habilitacyjne. Université Jagellon (Cracovie).

SOFFER O. 1985 - The Upper Palaeolithic of the Central Russian Plain. Academic Press (Orlando).
STREET M. \& TERBERGER Th. 1999 - The last Pleniglacial and the human settlement of Central Europe : new information from the Rhineland site of Wiesbaden-lgstadt. Antiquity 73 : 259-272.

SVOBODOVÁ H. 1991 - The pollen analysis of Dolní Vestonice II, section $\mathrm{n}^{\circ} 1$. In: Svoboda J. (Ed), Dolní Vestonice II Westem slope. ERAUL (Liège) 54: 75-88.

SVOBODA J. 2001 - Czech Republic: projects of the Center for Paleolithic and Paleoethnological Research (Institute of Archaeology, Academy of Sciences), BmoDolní Vestonice. In: Noiret P. (Ed), Le Paléolithique supérieur européen. Bilan quinquennal 1996-2001. ERAUL (Liège) 97: 73-88.

SVOBODOVÁ H. et SVOBODA J. 1988 - Chronostratigraphie et paléoécologie du Paléolithique supérieur morave d'après les fouilles récentes. Actes du Colloque "Cultures et industries paléolithiques en milieu loessique", Amiens, 9-11 décembre 1986. Revue archéologique de Picardie (Amiens) 1-2: 11-15.

SVOBODA J., CZUDEK T., HAVLÍCEK P., LOZEK V., MACOUN J., PRICHYSTAL A., SVOBODOVÁ H. \& VLCEK E. 1994 - Paleolit Moravy a Slezska. The Dolní Vestonice Studies, Institute of Archaeology, ASCR (Brno) 1.

SVOBODA J., KLÍMA B., JAROSOVÁ L. \& SKRDLA P. 2000 - The Gravettian in Moravia: climate, behaviour and technological complexity. In: Roebroeks W., Mussi M., Svoboda J. \& Fennema K. (Eds), Hunters of the Golden Age. The Mid Upper Palaeolithic of Eurasia 30,000 - 20,000 BP. University of Leiden (Leiden): 197-217.

VALOCH K. 1976 - Die altsteinzeitliche Fundstelle in Bmo-Bohunice. Brno : Ceskoslovenske Akademie ved (Studie Archeologickeho ustavu Ceskoslovenske Akademie ved v brne 4). 
